\title{
Cluster automorphism groups of cluster algebras with coefficients 1
}

\author{
Wen Chang and Bin Zhu \\ Department of Mathematical Sciences Department of Mathematical Sciences \\ Tsinghua University \\ 100084 Beijing, P. R. China \\ E-mail: changw12@mails.tsinghua.edu.cn \\ Tsinghua University \\ 100084 Beijing, P. R. China \\ E-mail: bzhu@math.tsinghua.edu.cn
}

\begin{abstract}
We study the cluster automorphism group of a skew-symmetric cluster algebra with geometric coefficients. For this, we introduce the notion of gluing free cluster algebra, and show that under a weak condition the cluster automorphism group of a gluing free cluster algebra is a subgroup of the cluster automorphism group of its principal part cluster algebra (i.e. the corresponding cluster algebra without coefficients). We show that several classes of cluster algebras with coefficients are gluing free, for example, cluster algebras with principal coefficients, cluster algebras with universal geometric coefficients, and cluster algebras from surfaces (except a 4-gon) with coefficients from boundaries. Moreover, except four kinds of surfaces, the cluster automorphism group of a cluster algebra from a surface with coefficients from boundaries is isomorphic to the cluster automorphism group of its principal part cluster algebra; for a cluster algebra with principal coefficients, its cluster automorphism group is isomorphic to the automorphism group of its initial quiver.
\end{abstract}

Key words. Cluster algebra; Cluster automorphism group; Gluing free cluster algebra; Cluster algebra from a surface; Universal geometric cluster algebra.

Mathematics Subject Classification. 16S99; 16S70; 18 E30

\section{Introduction}

After introduced by Sergey Fomin and Andrei Zelevinsky in [FZ02], cluster algebras have been showed to be linked to various areas of mathematics, see for examples, [GLS08, F10, L10, R10, K12, M14], and so on. However, as an algebra itself with combinatorical structure, it is natural and interesting to study the symmetries of a cluster algebra. For this, Assem, Schiffler and Shramchenko [ASS12] introduced cluster automorphisms and the cluster automorphism group of a cluster algebra without coefficients. These concepts and some similar ones are studied in many papers [S10, ASS12, BQ12, ASS13, BD13, KP13, N13, Z06]. In this paper, we initial the study of the cluster automorphism group of a cluster algebra with coefficients. We are interested in the dependence of the cluster automorphism group on the choice of coefficients. We are also interested in the relations between the cluster automorphism groups of different cluster algebras.

We consider in this paper the skew-symmetric cluster algebras of geometric type, that is, the cluster algebras determined by ice quivers without loops nor 2-cycles. An ice quiver is an oriented diagram $Q$ associated a subset $F=\{n+1, n+2, \cdots, n+m\}$ (the set of frozen vertices) of its vertex set $Q_{0}=\{1,2, \cdots, n+m\}$. The full subquiver of $Q$ with vertex set $Q_{0} \backslash F$ (the set of exchangeable vertices) is called the principal part of $Q$, and we denote it by $Q^{e x}$. By associating each vertex $1 \leqslant i \leqslant n+m$ of $Q$ an indeterminate element $x_{i}$, we have a set $\mathbf{x}=\left\{x_{1}, x_{2}, \cdots, x_{n+m}\right\}$, which

\footnotetext{
${ }^{1}$ Supported by the NSF of China (Grants 11131001)
} 
is called a cluster. Then the cluster algebra $\mathcal{A}_{Q}$ is a $\mathbb{Z}$-subalgebra of the rational function field $\mathcal{F}=\mathbb{Q}\left(x_{1}, \cdots, x_{n+m}\right)$ generated by variables in clusters obtained by iterated operations so called mutations from the initial cluster $\mathbf{x}$. The variables labeled by frozen vertices are the coefficients of $\mathcal{A}_{Q}$. The cluster algebra $\mathcal{A}_{Q^{e x}}$ is called the principal part cluster algebra of $\mathcal{A}_{Q}$, it is a coefficient free cluster algebra.

A cluster automorphism of $\mathcal{A}_{Q}$ is an algebra automorphism which maps the initial cluster $\mathbf{x}$ to a cluster and commutes with the mutation. An easy observation is that the group $\operatorname{Aut}\left(\mathcal{A}_{Q}\right) \operatorname{depends}$ on the choice of coefficients. To describe this more precisely, we classify the coefficients of $\mathcal{A}_{Q}$ by considering the relations of frozen vertices in $Q$ with respect to the exchangeable vertices. Let $j$ and $k$ be two frozen vertices, if the number of arrows from $j$ to $i$ (or from $i$ to $j$ ) is equal to the number of arrows from $k$ to $i$ (or from $i$ to $k$ ) for any exchangeable vertex $i$, then we say that these two vertices $j$ and $k$ are strictly glueable (Definition 3.1). It is not hard to see that exchanging the coefficients $x_{j}$ and $x_{k}$ induces a cluster automorphism of $\mathcal{A}_{Q}$ (see Proposition 2.5). The cluster algebra $\mathcal{A}_{Q}$ is called gluing free if there exist no frozen vertices are strictly glueable in $Q$ (see Definition 3.7). Then we prove that for a gluing free cluster algebra $\mathcal{A}_{Q}$ with at least two exchangeable vertices in $Q$, the group $\operatorname{Aut}\left(\mathcal{A}_{Q}\right)$ is a subgroup of $\operatorname{Aut}\left(\mathcal{A}_{Q^{e x}}\right.$ ) (see Theorem 3.16).

To prove Theorem 3.16, we consider the exchange graph $E_{Q}$ of $\mathcal{A}_{Q}$, which is a n-regular connected graph whose vertices are clusters and whose edges are labeled by mutations. We introduce the automorphism group $A u t\left(E_{Q}\right)$ of $E_{Q}$ which consisting of automorphisms as a graph 2 . Then by using the fact that $E_{Q}$ is independent on the choice of coefficients[CKLP13], we prove Theorem 3.16 by viewing $\operatorname{Aut}\left(\mathcal{A}_{Q}\right)$ and $\operatorname{Aut}\left(\mathcal{A}_{Q^{e x}}\right)$ as subgroups of $\operatorname{Aut}\left(E_{Q}\right)$.

In this paper, three kinds of cluster algebras are of particular importance. The first one is the universal geometric cluster algebra (Definition 2.7), which is slightly different from the initial universal cluster algebra introduced in [FZ07]. It is a universal object, in the view point of coefficient specialization, in the set of cluster algebras with the same principal part. The second one is the cluster algebra arising from an oriented marked Reimman surface with boundary[[FST08]. The another kind is a cluster algebra with principal coefficients. We show that a cluster algebra with universal geometric coefficients, a cluster algebra (with coefficients) from a surface (except a 4-gon), and a cluster algebra with principal coefficients are gluing free in Proposition 3.9, Proposition 3.10 and Proposition 3.8 respectively. Thus by Theorem 3.16 the automorphism groups of these cluster algebras are all the subgroups of the corresponding principal part cluster algebras.

Generally, for a gluing free cluster algebra $\mathcal{A}_{Q}, \operatorname{Aut}\left(\mathcal{A}_{Q}\right)$ may be a proper subgroup of $\operatorname{Aut}\left(\mathcal{A}_{Q^{e x}}\right)$ (see Example 7). But for the cluster algebras from oriented marked Riemann surfaces, these two groups are isomorphic with each other (Theorem 3.18). For a cluster algebra with principal coefficients, the cluster automorphism group is isomorphic to the automorphism group of the initial quiver (Theorem 3.19).

As showed in Example 7, for an universal geometric cluster algebra $\mathcal{A}^{\text {univ }}$ with principal part $\mathcal{A}$, $\operatorname{Aut}\left(\mathcal{A}^{\text {univ }}\right)$ may be a proper subgroup of $\operatorname{Aut}(\mathcal{A})$. However, we prove in the subsequent paper [CZ15a] that $\operatorname{Aut}\left(\mathcal{A}^{\text {univ }}\right)$ is always isomorphic to $\operatorname{Aut}(\mathcal{A})$, if $\mathcal{A}$ is a cluster algebra of finite type

\footnotetext{
${ }^{2}$ We owe this definition to Thomas Brüstle.
} 
and $\mathcal{A}^{\text {univ }}$ is the $F Z$-universal cluster algebra introduced in [FZ07]. Generally, $\operatorname{Aut}\left(\mathcal{A}_{Q}\right)$ may be a proper subgroup of $\operatorname{Aut}\left(E_{Q}\right)$, even though $\mathcal{A}_{Q}$ is coefficient free. In the subsequent paper [CZ15b] we prove that for a coefficient free cluster algebra $\mathcal{A}_{Q}$, the two groups $\operatorname{Aut}\left(\mathcal{A}_{Q}\right)$ and $\operatorname{Aut}\left(E_{Q}\right)$ are isomorphic, if $\mathcal{A}$ is of finite type, excepting types of rank two and type $F_{4}$, or $\mathcal{A}$ is of skew-symmetric finite mutation type, that is, a cluster algebra with only finite quivers up to isomorphisms.

The paper is organized as follows. In section 2, we recall some basic notions on cluster algebras, especially cluster automorphisms, coefficient specializations and cluster algebras arising from surfaces. In subsection 3.1 we define the notion of gluing free cluster algebra and prove some properties. We introduce and consider the automorphism group of an exchange graph in subsection 3.2. Finally, in subsection 3.3 we prove some main results.

\section{Preliminaries}

\subsection{Cluster algebras}

We recall that a quiver is a quadruple $\left(Q_{0}, Q_{1}, s, t\right)$ consisting of a set of vertices $Q_{0}$, of a set of arrows $Q_{1}$, and of two maps $s, t$ which map each arrow $\alpha \in Q_{1}$ to its source $s(\alpha)$ and its target $t(\alpha)$, respectively. An ice quiver is a quiver $Q$ associated a subset $F$ (the set of frozen vertices) of $Q_{0}$. The full subquiver $Q^{e x}$ of $Q$ with vertex set $Q_{0} \backslash F$ (the set of exchangeable vertices) is called the principal part of $Q$. The opposite quiver of $Q$ is a quiver $Q^{o p}$ obtained from $Q$ by reversing all the arrows. We always assume in this paper that there are no loops nor 2-cycles in an ice quiver, and no arrows between frozen vertices. We also assume that an ice quiver and its principal part are connected. For two ice quivers $Q$ and $Q^{\prime}$, an isomorphism $\sigma$ from $Q$ to $Q^{\prime}$ is a bijection from $Q_{0}$ to $Q_{0}^{\prime}$, which maps exchangeable vertices (and frozen vertices) to exchangeable ones (and frozen ones), such that the number of arrows from a vertex $i$ to a vertex $j$ in $Q_{0}$ is equal to the number of arrows from $\sigma(i)$ to $\sigma(j)$ in $Q_{0}^{\prime}$, we write $Q \cong Q^{\prime}$ to quivers which are isomorphic with each other. Then for a quiver $Q$, all of its automorphisms consist a group $\operatorname{Aut}(Q)$, we call it the automorphism group of $Q$.

Let $n+m=\left|Q_{0}\right|$ be the number of vertices in $Q$, and denote the vertices by $Q_{0}=\{1,2, \cdots, n+m\}$ and the frozen vertices by $F=\{n+1, n+2, \cdots, n+m\}$. We associate an extended skew-symmetric matrix $B=\left(b_{j i}\right)_{(n+m) \times n}$ to $Q$, where

$$
b_{j i}= \begin{cases}\sharp\{\alpha: j \rightarrow i \text { in } Q\} & \text { if there exist arrows from } \mathrm{j} \text { to } \mathrm{i} \text { in } \mathrm{Q} ; \\ -\sharp\{\alpha: i \rightarrow j \text { in } Q\} & \text { if there exist arrows from } \mathrm{i} \text { to } \mathrm{j} \text { in } \mathrm{Q} ; \\ 0 & \text { if there exist no arrows between } \mathrm{i} \text { and } \mathrm{j} \text { in } \mathrm{Q} .\end{cases}
$$

We call $B$ the exchange matrix of $Q$, its upper $n \times n$ part the principal part of $B$ and its lower $m \times n$ part the frozen part of $B$. Let $i$ be an exchangeable vertex of $Q$, we define a mutation of $Q$ and a mutation of $B$ as follows.

Definition 2.1. [FZ02]

(a) The mutation of $Q$ at $i$ is an ice quiver $\mu_{i}(Q, F)=\left(\mu_{i}(Q), \mu_{i}(F)\right)$, where $\mu_{i}(F)=F$ and $\mu_{i}(Q)$ is obtained from $Q$ by: 
- inserting a new arrow $\gamma: j \rightarrow k$ for each path $j \stackrel{\alpha}{\rightarrow} i \stackrel{\beta}{\rightarrow} k$;

- inverting all arrows passing through $i$;

- removing the arrows in a maximal set of pairwise disjoint 2-cycles;

- removing the arrows between frozen vertices.

(b) The mutation of $B$ at $i$ is a matrix $\mu_{i}(B)=\left(b_{j k}^{\prime}\right)_{n \times n} \in M_{n \times n}(\mathbb{Z})$ given by

$$
b_{j k}^{\prime}= \begin{cases}-b_{j k} & \text { if } k=i \text { or } j=i \\ b_{j k}+\frac{1}{2}\left(\left|b_{j k}\right| b_{k i}+b_{j k}\left|b_{k i}\right|\right) & \text { otherwise. }\end{cases}
$$

Then these two kinds of mutations correspond with each other, that is, $\mu_{i}(B)$ is the exchange matrix of $\mu_{i}(Q)$. We always write $\mu_{i}(Q)$ to $\mu_{i}(Q, F)$ for brevity. By associating each vertex $1 \leqslant i \leqslant n+m$ in $Q_{0}$ an indeterminate element $x_{i}$, we have a set $\mathbf{x}=\left\{x_{1}, x_{2}, \cdots, x_{n+m}\right\}$. We call $\Sigma=(Q, \mathbf{x})$ a seed, $\mathbf{x}$ a cluster and an element in $\mathbf{x}$ a cluster variable.

Definition 2.2. [FZ02 Let $i$ be an exchangeable vertex of $Q$, the mutation of the seed $\Sigma$ in the direction $i\left(\right.$ or $\left.x_{i}\right)$ is a seed $\mu_{i}(\Sigma)=\left(\mu_{i}(Q), \mu_{i}(\mathbf{x})\right)$, where $\mu_{i}(\mathbf{x})=\left(\mathbf{x} \backslash\left\{x_{i}\right\}\right) \sqcup\left\{x_{i}^{\prime}\right\}$ and:

$$
x_{i} x_{i}^{\prime}=\prod_{\substack{1 \leqslant j \leqslant n ; \\ b_{j i}>0}} x_{j}^{b_{j i}}+\prod_{\substack{1 \leqslant j \leqslant n ; \\ b_{j i}<0}} x_{j}^{-b_{j i}}
$$

We call (1) the exchange relation of the mutation, and call

$$
p_{i}^{+}=\prod_{\substack{1 \leqslant j \leqslant n ; \\ b_{j i}>0}} x_{j}^{b_{j i}} \quad \text { and } \quad p_{i}^{-}=\prod_{\substack{1 \leqslant j \leqslant n ; \\ b_{j i}<0}} x_{j}{ }^{-b_{j i}}
$$

the coefficients of the exchange relation.

It is easy to check that a seed mutation is an involution, that is $\mu_{i} \mu_{i}(\Sigma)=\Sigma$.

Definition 2.3. [FZ02] Denote by $\mathscr{X}$ the union of all possible clusters obtained from $\mathbf{x}$ by iterated mutations. The cluster algebra $\mathcal{A}_{Q}$ is a $\mathbb{Z}$-subalgebra of the rational function field $\mathcal{F}=\mathbb{Q}\left(x_{1}, \cdots\right.$ $\left.\cdot, x_{n+m}\right)$ generated by cluster variables in $\mathscr{X}$.

We call the variables in $\mathbf{f} \mathbf{x}=\left\{x_{n+1}, \ldots, x_{n+m}\right\}$ the frozen cluster variables of $\mathcal{A}_{Q}$, and call the rest variables in $\mathscr{X}$ the exchangeable cluster variables of $\mathcal{A}_{Q}$. Then in a seed $(\tilde{Q}, \tilde{\mathbf{x}}), \tilde{\mathbf{x}}=\tilde{\mathbf{e x}} \sqcup \mathbf{f} \mathbf{x}$, where $\tilde{\mathbf{e x}}$ is the set of exchangeable cluster variables. Let $\mathbb{P}$ be the coefficient group of $\mathcal{A}_{Q}$, which is a multiplicative free abelian group generated by elements in $\mathbf{f x}$. We call the elements in $\mathbb{P}$ the coefficients of $\mathcal{A}_{Q}$. We denote by $\mathcal{A}_{Q}\left[\mathbb{P}^{-1}\right]$ the localization of $\mathcal{A}_{Q}$ at $\mathbb{P}$, which is called the localized cluster algebra of $\mathcal{A}_{Q}$. Note that $\mathcal{A}_{Q}\left[\mathbb{P}^{-1}\right]$ is the initial definition of the geometric cluster algebras given in [FZ02]. A sequence $\left(y_{1}, \cdots, y_{l}\right)$ is called $(Q, \mathbf{x})$-admissible if $y_{1}$ is exchangeable in $\mathbf{x}$ and $y_{i}$ is exchangeable in $\mu_{y_{i-1}} \circ \cdots \circ \mu_{y_{1}}(\mathbf{x})$ for every $2 \leqslant i \leqslant l$. A rooted cluster algebra associated to $\mathcal{A}_{Q}$ is a triple $\left(Q, \mathbf{x}, \mathcal{A}_{Q}\right)$ [ADS14]. We call the pair $(Q, \mathbf{x})$ the initial seed and $\mathbf{x}$ the initial cluster. We strictly distinguish the cluster algebras and the rooted cluster algebras in the rest of the paper. It has been shown in [GSV08] that a cluster $\tilde{\mathbf{x}}$ determines the quiver $\tilde{Q}$, and we denote its quiver by $Q(\tilde{\mathbf{x}})$ and write $p_{x}$ to the vertex of $\tilde{Q}$ labeled by a variable $x$ in $\tilde{\mathbf{x}}$. 


\subsection{Cluster automorphisms}

Let $\left(Q, \mathbf{x}, \mathcal{A}_{Q}\right)$ and $\left(Q^{\prime}, \mathbf{x}^{\prime}, \mathcal{A}_{Q^{\prime}}\right)$ be two rooted cluster algebras, where $\mathbf{x}=\mathbf{e x} \sqcup \mathbf{f} \mathbf{x}$ and $\mathbf{x}^{\prime}=$ $\mathbf{e x}^{\prime} \sqcup \mathbf{f x}^{\prime}$. Recall from [ADS14] that a rooted cluster morphism $f$ from $\left(Q, \mathbf{x}, \mathcal{A}_{Q}\right)$ to $\left(Q^{\prime}, \mathbf{x}^{\prime}, \mathcal{A}_{Q^{\prime}}\right)$ is a ring homomorphism $f: \mathcal{A}_{Q} \rightarrow \mathcal{A}_{Q^{\prime}}$ satisfies the following three conditions,

(CM1) $f(\mathbf{e x}) \subset \mathbf{e x}^{\prime} \sqcup \mathbb{Z}$;

(CM2) $f(\mathbf{f x}) \subset \mathbf{x}^{\prime} \sqcup \mathbb{Z}$;

(CM3) for every $\left(f, \mathbf{x}, \mathbf{x}^{\prime}\right)$-biadmissible sequence $\left(y_{1}, \cdots, y_{l}\right)$ and any $y$ in $\mathbf{x}$, we have $f\left(\mu_{y_{l}} \circ\right.$ $\left.\cdots \circ \mu_{y_{1}, \mathbf{x}}(y)\right)=\mu_{f\left(y_{l}\right)} \circ \cdots \circ \mu_{f\left(y_{1}\right), \mathbf{x}^{\prime}}(f(y))$. Here a $\left(f, \mathbf{x}, \mathbf{x}^{\prime}\right)$-biadmissible sequence $\left(y_{1}, \cdots, y_{l}\right)$ is a $(Q, \mathbf{x})$-admissible sequence such that $\left(f\left(y_{1}\right), \cdots, f\left(y_{l}\right)\right)$ is $\left(Q^{\prime}, \mathbf{x}^{\prime}\right)$-admissible.

Definition 2.4. Let $\mathcal{A}$ and $\mathcal{A}^{\prime}$ be two cluster algebras.

(a) We call a map $f: \mathcal{A}^{\prime} \rightarrow \mathcal{A}$ a cluster isomorphism if there exist two seeds $\left(Q^{\prime}, \mathbf{x}^{\prime}\right)$ and $(Q, \mathbf{x})$ of $\mathcal{A}^{\prime}$ and $\mathcal{A}$ respectively, and a bijective rooted cluster morphism $\tilde{f}$ from $\left(Q^{\prime}, \mathbf{x}^{\prime}, \mathcal{A}^{\prime}\right)$ to $(Q, \mathbf{x}, \mathcal{A})$ such that $f=\tilde{f}$ on $\mathcal{A}^{\prime}$.

(b) We call $f: \mathcal{A} \rightarrow \mathcal{A}$ a cluster automorphism if $f$ is a cluster isomorphism.

(c) We call Aut $(\mathcal{A})$ the cluster automorphism group of $\mathcal{A}$, which is a group consisting of cluster automorphisms of $\mathcal{A}$ under compositions of automorphisms.

The cluster automorphism of a coefficient free cluster algebra and its equivalent characterizations is given in [ASS12]. For cluster algebras with coefficients, we also have the following characterizations.

Proposition 2.5. A $\mathbb{Z}$-algebra automorphism $f: \mathcal{A} \rightarrow \mathcal{A}$ is a cluster automorphism if and only if one of the following conditions is satisfied:

1. there exists a seed $\Sigma=(\mathbf{e x}, \mathbf{f} \mathbf{x}, Q)$ of $\mathcal{A}$, such that $f(\mathbf{x})$ is the cluster in a seed $\Sigma^{\prime}=$ $\left(\mathbf{e x}^{\prime}, \mathbf{f x}^{\prime}, Q^{\prime}\right)$ of $\mathcal{A}$ with $Q^{\prime} \cong Q$ or $Q^{\prime} \cong Q^{o p}$, under the correspondence $p_{x} \mapsto p_{f(x)}$;

2. for every seed $\Sigma=(\mathbf{e x}, \mathbf{f x}, Q)$ of $\mathcal{A}, f(\mathbf{x})$ is the cluster in a seed $\Sigma^{\prime}=\left(\mathbf{e x}^{\prime}, \mathbf{f x}^{\prime}, Q^{\prime}\right)$ with $Q^{\prime} \cong Q$ or $Q^{\prime} \cong Q^{o p}$, under the correspondence $p_{x} \mapsto p_{f(x)}$.

Proof. Since $Q$ and its principal part are both connected, the proofs are similar to the proofs of Lemma 2.3 and Proposition 2.4 in [ASS12].

Lemma 2.6. We call a cluster automorphism of $\mathcal{A}_{Q}$ which maps $Q$ to a quiver isomorphic to $Q$ a direct cluster automorphism, then all the direct cluster automorphisms consist a normal subgroup $\operatorname{Aut}^{+}\left(\mathcal{A}_{Q}\right)$ of $\operatorname{Aut}(\mathcal{A})$ with the index at most two.

Proof. By using above proposition, the proof is similar to the proof of Lemma 2.9 in [ASS12].

\subsection{Coefficient specializations}

The cluster algebra with universal coefficients is defined in [FZ07], we state its definition in our settings, that is, the universal geometric cluster algebra. 
Definition 2.7. [FZ07 R14a] Let $Q$ be a quiver with $n$ exchangeable vertices and without frozen vertices. We denote by $R C A(Q)$ the set of rooted cluster algebras $\left(\tilde{Q}, \tilde{\mathbf{x}}, \mathcal{A}_{\tilde{Q}}\right)$, where $\mathcal{A}_{\tilde{Q}}$ are cluster algebras of the ice quivers with the principal part $Q$.

(a) Let $\left(Q^{\prime}, \mathbf{x}^{\prime}, \mathcal{A}_{Q^{\prime}}\right)$ and $\left(Q^{\prime \prime}, \mathbf{x}^{\prime \prime}, \mathcal{A}_{Q^{\prime \prime}}\right)$ be two rooted cluster algebras in $R C A(Q)$. Let $\mathbb{P}^{\prime}$ and $\mathbb{P}^{\prime \prime}$ be the coefficient groups of $\mathcal{A}_{Q^{\prime}}$ and $\mathcal{A}_{Q^{\prime \prime}}$ respectively. If there is a group homomorphism $\varphi: \mathbb{P}^{\prime} \rightarrow \mathbb{P}^{\prime \prime}$ that extends to a ring homomorphism $\varphi: \mathcal{A}_{Q^{\prime}}\left[\mathbb{P}^{\prime-1}\right] \rightarrow \mathcal{A}_{Q^{\prime \prime}}\left[\mathbb{P}^{\prime \prime-1}\right]$ such that $\varphi\left(\mu_{\varpi}\left(x_{i}^{\prime}\right)\right)=\mu_{\varpi}\left(x_{i}^{\prime \prime}\right)$ for any $1 \leqslant i \leqslant n$ and any admissible sequence $\varpi$, we say that both $\varphi: \mathbb{P}^{\prime} \rightarrow \mathbb{P}^{\prime \prime}$ and $\varphi: \mathcal{A}_{Q^{\prime}}\left[\mathbb{P}^{\prime-1}\right] \rightarrow \mathcal{A}_{Q^{\prime \prime}}\left[\mathbb{P}^{\prime \prime-1}\right]$ are coefficient specializations.

(b) Let $\left(Q^{\text {univ }}, \mathbf{x}^{\text {univ }}, \mathcal{A}_{Q^{\text {univ }}}\right)$ be a rooted cluster algebra in $R C A(Q)$ with coefficient group $\mathbb{P}$. We call $\mathcal{A}_{Q^{\text {univ }}}$ a universal geometric cluster algebra of $R C A(Q)$, if for any rooted cluster algebra $\left(Q^{\prime}, \mathbf{x}^{\prime}, \mathcal{A}_{Q^{\prime}}\right)$ in $R C A(Q)$ with coefficient group $\mathbb{P}^{\prime}$, there is a unique coefficient specialization from $\mathcal{A}_{Q^{\text {univ }}}\left[\mathbb{P}^{-1}\right]$ to $\mathcal{A}_{Q^{\prime}}\left[\mathbb{P}^{\prime-1}\right]$.

Remark 2.8. Since the cluster algebras we considered are geometric ones, the universal geometric cluster algebra is different from the initial cluster algebra with universal coefficients given in [FZ02]. Some deformed universal geometric cluster algebras and their existences have been considered in a series of papers [R12] R14a R14b].

The following is an equivalent characterization of coefficient specializations.

Lemma 2.9. [FZ07] Let $\left(Q^{\prime}, \mathbf{x}^{\prime}, \mathcal{A}_{Q^{\prime}}\right)$ and $\left(Q^{\prime \prime}, \mathbf{x}^{\prime \prime}, \mathcal{A}_{Q^{\prime \prime}}\right)$ be two rooted cluster algebras in $R C A(Q)$. Let $\mathbb{P}^{\prime}$ and $\mathbb{P}^{\prime \prime}$ be the coefficient groups of $\mathcal{A}_{Q^{\prime}}$ and $\mathcal{A}_{Q^{\prime \prime}}$ respectively. A group homomorphism $\varphi: \mathbb{P}^{\prime} \rightarrow \mathbb{P}^{\prime \prime}$ is a coefficient specialization if and only if

$$
\varphi\left(p_{i, \varpi}^{\prime \pm}\right)=p_{i, \varpi}^{\prime \prime \pm}
$$

for any exchangeable vertex $i$ and admissible sequence $\varpi$, where $p_{i, \varpi}^{\prime \pm}$ and $p_{i, \varpi}^{\prime \prime \pm}$ are coefficients in exchange relations of $\left(\mu_{\varpi}\left(Q^{\prime}\right), \mu_{\varpi}\left(\mathbf{x}^{\prime}\right)\right)$ and of $\left(\mu_{\varpi}\left(Q^{\prime \prime}\right), \mu_{\varpi}\left(\mathbf{x}^{\prime \prime}\right)\right)$ respectively.

It follows from [FZ07] that a cluster algebra with universal coefficients (if there exists one) is unique. In our settings, we have the following more specific characterization for the uniqueness of the universal geometric cluster algebras.

Lemma 2.10. Let $\left(Q^{\prime}, \mathbf{x}^{\prime}, \mathcal{A}_{Q^{\prime}}\right)$ and $\left(Q^{\prime \prime}, \mathbf{x}^{\prime \prime}, \mathcal{A}_{Q^{\prime \prime}}\right)$ be two rooted cluster algebras in $R C A(Q)$ with $\mathcal{A}_{Q^{\prime}}$ and $\mathcal{A}_{Q^{\prime \prime}}$ the universal geometric cluster algebras. We denote by $B=\left(b_{j i}\right)_{n \times n}$ the exchange matrix corresponding to $Q$, and by $\left.\left(\begin{array}{c}B \\ B^{\prime}\end{array}\right)=\left(b_{j i}^{\prime}\right)_{\left(n+m^{\prime}\right.}\right) \times n\left(\right.$ or $\left(\begin{array}{c}B \\ B^{\prime \prime}\end{array}\right)=\left(b_{j i}^{\prime \prime}\right)_{\left(n+m^{\prime \prime}\right) \times n}$ respectively) the exchange matrix corresponding to $Q^{\prime}$ (or $Q^{\prime \prime}$ respectively). Then $m^{\prime}=m^{\prime \prime}$ and there is an invertible matrix $A=\left(a_{j k}\right)_{m^{\prime} \times m^{\prime \prime}}$ with entries in $\mathbb{Z}$ such that

$$
A^{t} \mu_{\varpi}\left(B^{\prime}\right)=\mu_{\varpi}\left(B^{\prime \prime}\right)
$$

for any admissible sequence $\varpi$, where $\mu_{\varpi}\left(B^{\prime}\right)$ and $\mu_{\varpi}\left(B^{\prime \prime}\right)$ are frozen parts of $\mu_{\varpi}\left(\begin{array}{c}B \\ B^{\prime}\end{array}\right)$ and $\mu_{\varpi}\left(\begin{array}{c}B \\ B^{\prime \prime}\end{array}\right)$ respectively.

Proof. Let $\mathbb{P}^{\prime}$ and $\mathbb{P}^{\prime \prime}$ be the coefficient groups of $\mathcal{A}_{Q^{\prime}}$ and $\mathcal{A}_{Q^{\prime \prime}}$ respectively. Since $\mathcal{A}_{Q^{\prime}}$ and $\mathcal{A}_{Q^{\prime \prime}}$ are both universal geometric cluster algebras with the same principal part, there are two coefficient specializations $\varphi: \mathbb{P}^{\prime} \rightarrow \mathbb{P}^{\prime \prime}$ and $\psi: \mathbb{P}^{\prime \prime} \rightarrow \mathbb{P}^{\prime}$. Then the composition $\psi \varphi$ is a 
coefficient specialization from $\mathbb{P}^{\prime}$ to itself and thus $\psi \varphi$ is the identity. Since $\mathbb{P}^{\prime}$ and $\mathbb{P}^{\prime \prime}$ are both free abelian groups, they have the same rank, thus $m^{\prime}=m^{\prime \prime}$. We assume that

$$
\varphi\left(x_{n+j}^{\prime}\right)=\prod_{1 \leqslant k \leqslant m^{\prime \prime}} x_{n+k}^{\prime \prime} a_{j k}
$$

for any $1 \leqslant j \leqslant m^{\prime}$, where each $a_{j k}$ is an integer number. Then we have a matrix $A=\left(a_{j k}\right)$ which is invertible since $\varphi$ is an isomorphism. For any $1 \leqslant i \leqslant n$, we consider the exchange relations

$$
\mu_{i}\left(x_{i}^{\prime}\right) x_{i}^{\prime}=\prod_{b_{j i}^{\prime}>0} x_{j}^{\prime b_{j i}^{\prime}}+\prod_{b_{j i}^{\prime}<0} x_{j}^{\prime-b_{j i}^{\prime}}
$$

and

$$
\mu_{i}\left(x_{i}^{\prime \prime}\right) x_{i}^{\prime \prime}=\prod_{b_{k i}^{\prime \prime}>0} x_{k}^{\prime \prime \prime} b_{k i}^{\prime \prime}+\prod_{b_{k i}^{\prime \prime}<0} x_{k}^{\prime \prime \prime} b_{k i}^{\prime \prime} .
$$

Since $\varphi\left(x_{j}^{\prime}\right)=x_{j}^{\prime \prime}$ for any $1 \leqslant j \leqslant n$, the equality $\varphi\left(\mu_{i}\left(x_{i}^{\prime}\right)=\mu_{i}\left(x_{i}^{\prime \prime}\right)\right.$ is equivalent to

$$
\begin{gathered}
\prod_{\substack{1 \leqslant j \leqslant m^{\prime} \\
b_{j i}^{\prime}>0}}\left(\prod_{1 \leqslant k \leqslant m^{\prime \prime}} x_{n+k}^{\prime \prime} a_{j k}\right)^{b_{(n+j) i}^{\prime}}=\prod_{\substack{1 \leqslant j \leqslant m^{\prime \prime} \\
b_{j i}^{\prime \prime}>0}} x_{n+k}^{\prime \prime} b_{(n+k) i}^{\prime \prime} \text { and } \\
\prod_{\substack{1 \leqslant j \leqslant m^{\prime} \\
b_{j i}^{\prime}<0}}\left(\prod_{1 \leqslant k \leqslant m^{\prime \prime}} x_{n+k}^{\prime \prime} a_{j k}\right)^{-b_{(n+j) i}^{\prime}}=\prod_{\substack{1 \leqslant j \leqslant m^{\prime \prime} \\
b_{j i}^{\prime \prime}<0}} x_{n+k}^{\prime \prime}-b_{(n+k) i}^{\prime \prime} .
\end{gathered}
$$

Thus we have

$$
\prod_{1 \leqslant j \leqslant m^{\prime}}\left(\prod_{1 \leqslant k \leqslant m^{\prime \prime}} x_{n+k}^{\prime \prime} a_{j k}\right)^{b_{(n+j) i}^{\prime}}=\prod_{1 \leqslant k \leqslant m^{\prime \prime}} x_{n+k}^{\prime \prime} b_{(n+k) i}^{\prime \prime},
$$

and finally

$$
\sum_{1 \leqslant k \leqslant m^{\prime \prime}} a_{j k} b_{(n+j) i}^{\prime}=b_{(n+k) i}^{\prime \prime}
$$

Therefore we have $A^{t} B^{\prime}=B^{\prime \prime}$. By induction, we can prove the general case $A^{t} \mu_{\varpi}\left(B^{\prime}\right)=\mu_{\varpi}\left(B^{\prime \prime}\right)$ for any admissible sequence $\varpi$.

The existence of universal geometric cluster algebras is not clearly at all. A cluster algebra of finite type with universal coefficients is given in [FZ02]. It is a geometric cluster algebra, and its frozen rows are given by $g$-vectors of the corresponding cluster algebra with principal coefficients, we call it $F Z$-universal cluster algebra.

Example 1. We consider the FZ-universal cluster algebra $\mathcal{A}_{Q^{\prime}}$ of type $A_{2}$, whose quiver $Q^{\prime}$ is as follows with matrix $\left(\begin{array}{l}B \\ B^{\prime}\end{array}\right)$, where 1 and 2 are exchangeable vertices and the others are frozen ones.

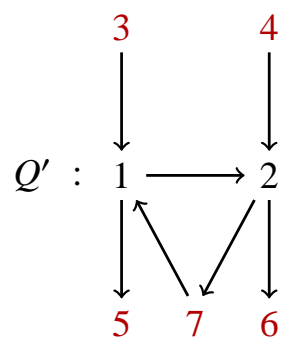


Given the following invertible matrix $A$, denote by $Q^{\prime \prime}$ the ice quiver of $\left(\begin{array}{c}B \\ B^{\prime \prime}\end{array}\right)$, where $B^{\prime \prime}=A^{t} B^{\prime}$.

$$
A:\left(\begin{array}{lllll}
2 & 1 & 0 & 0 & 0 \\
1 & 1 & 0 & 0 & 0 \\
0 & 0 & 3 & 2 & 0 \\
0 & 0 & 1 & 1 & 0 \\
0 & 0 & 0 & 0 & 1
\end{array}\right)
$$

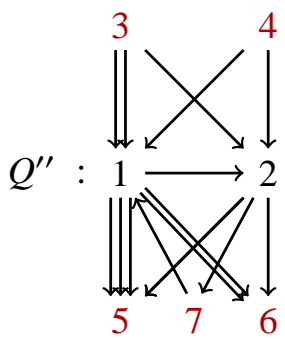

$A$

Then as the construction in above lemma, the matrix $A$ induces a group homomorphism $\varphi$ from the coefficient group $\mathbb{P}^{\prime}$ of $\mathcal{A}_{Q^{\prime}}$ to the coefficient group $\mathbb{P}^{\prime \prime}$ of $\mathcal{A}_{Q^{\prime \prime}}$, and it is straightforward to check that $\varphi$ is a coefficient specialization. Thus $\mathcal{A}_{Q^{\prime \prime}}$ is another universal cluster algebra of type $A_{2}$, which is not a FZ-universal cluster algebra. We will show in Example 7 that the cluster automorphism group $A u t\left(\mathcal{A}_{Q^{\prime}}\right)$ is $D_{5}$ which is the biggest one in the cluster automorphism groups of gluing free cluster algebras of type $A_{2}$, while Aut $\left(\mathcal{A}_{Q^{\prime \prime}}\right)$ is $\{i d\}$ which is the smallest one.

\subsection{Cluster algebras from surfaces}

Following [FST08], we recall oriented marked Riemann surfaces and the cluster algebras arising from surfaces. An oriented marked Riemann surface (or surface for brevity) is a pair $(S, M)$, where $S$ is a connected oriented Riemann surface with boundary and $M$ is a finite set of marked points on $S$. The marked points in the interior of $S$ are called punctures. We assume that $S$ is not closed and there is at least one marked point on each connected component of the boundary. We always assume that, for some technical reasons, $(S, M)$ is none of a disc with one, two or three marked points on the boundary; or a once-punctured disc with one marked point on the boundary.

Two curves in $(S, M)$ are the same if they are isotopic with respect to marked points. Two curves $\gamma$ and $\gamma^{\prime}$ are compatible if there are representatives of $\gamma$ and $\gamma^{\prime}$ which do not intersect in $S \backslash$ $M$. An arc is a non-contracted curve, with endpoints in $M$ which are the only possible selfintersection points. An arc is a boundary arc if it is isotopic to a boundary component with respect to marked points, otherwise it is an internal arc. An ideal triangulation of $(S, M)$ is a maximal collection of compatible internal arcs. An ideal triangulation of $(S, M)$ with boundary[ST09, ADS14 is a maximal collection of compatible arcs (which contains the boundary arcs). The arcs of the triangulation cut the surface into triangles (which may be self-folded). Given an ideal triangulation $T$ without self-folded triangles (see Section 4 in [FST08] for the general case), we associate to it a quiver $Q_{T}$ as follows.

(a) the vertices in $Q_{T}$ are the $\operatorname{arcs}$ in $T$;

(b) if two arcs $\gamma$ and $\gamma^{\prime}$ are both sides of a triangle and $\gamma^{\prime}$ follows $\gamma$ in the positive direction, then add an arrow from $\gamma$ to $\gamma^{\prime}$;

(c) deleting all the 2-cycles.

For the corresponding ideal triangulation $T^{b}$ with boundary, we associate to it an ice quiver $Q_{T^{b}}$ in a similar way (we delete the arrows between boundary arcs), where the frozen vertices are the 
boundary arcs. Then $Q_{T}$ is the principal part of $Q_{T^{b}}$. We associate cluster algebras $\mathcal{A}_{Q_{T}}$ and $\mathcal{A}_{Q_{T^{b}}}$ to $Q_{T}$ and $Q_{T^{b}}$ respectively. It is proved in [FST08] that the cluster algebras $\mathcal{A}_{Q_{T}}$ and $\mathcal{A}_{Q_{T^{b}}}$ are independent on the choice of the triangulations. We call them the cluster algebras of $(S, M)$, without boundary and with boundary respectively, and denote them by $\mathcal{A}(S, M)$ and $\mathcal{A}^{b}(S, M)$ respectively. We obtain a new triangulation by flipping an ideal triangulation without self-folded triangles, where the flip of the triangulation corresponds to the mutation of the quiver. To flip an ideal triangulation at the internal arc of a self-folded triangle, Fomin, Shapiro and Thurston [FST08] introduced the tagged triangulation by adding tags 0 or 1 on the punctures. These triangulations one-to-one correspond to the clusters of $\mathcal{A}_{Q_{T}}$ and induce one-to-one correspondences from the tagged arcs to the cluster variables. It is showed in [FST08] that any ideal triangulation, except for a triangulation of a 4-punctured sphere (which is closed and thus eliminated in our assumptions of the surfaces), can be obtained by gluing the puzzle pieces in Figure 1 along the matched sides (see Section 4 of [FST08] for details). The following lemma is useful in the paper.
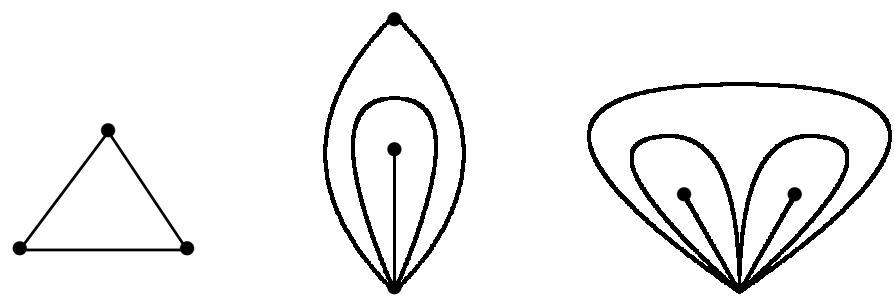

Figure 1: The three puzzle pieces

Lemma 2.11. [FST08](Lemma 2.13) The surface $(S, M)$ has an ideal triangulation which contains no self-folded triangles.

For a surface $(S, M)$, the marked mapping class group describes the symmetries of the surface $S$ with respect to the marked points and the tags of the punctures. We recall its definition from [ASS12]. We denote by $\operatorname{Homeo}^{+}(S, M)$ the group of orientation-preserving homeomorphisms from $S$ to $S$ that fix $M$ setwise, and $\operatorname{Homeo}_{0}(S, M)$ the subgroup of $\operatorname{Homeo}^{+}(S, M)$ which consisting of homeomorphisms that are isotopic to the identity relative to $M$. Then the mapping class group $\mathcal{M G}(S, M)$ is defined as the quotient

$$
\mathcal{M G}(S, M)=\operatorname{Homeo}^{+}(S, M) / \operatorname{Homeo}_{0}(S, M) .
$$

Note that the group $\mathcal{M G}(S, M)$ acts on the set of ideal triangulations in the natrual way. Let $\mathcal{Z}$ be the power set of the set of punctures of $(S, M)$. A homeomorphism in $\operatorname{Homeo}^{+}(S, M)$ acts on $\mathcal{Z}$ pointwise. The set $\mathcal{Z}$ is a group with respect to the operation $\ominus: \mathcal{P}_{1} \ominus \mathcal{P}_{2}=\left(\mathcal{P}_{1} \cup \mathcal{P}_{2}\right) \backslash\left(\mathcal{P}_{1} \cap \mathcal{P}_{2}\right)$. We define the marked mapping class group $\mathcal{M} \mathcal{G}_{\bowtie}(S, M)$ of the surface $(S, M)$ to be the semidirect product

$$
\mathcal{M} \mathcal{G}_{\bowtie}(S, M)=\mathcal{Z} \rtimes \mathcal{M G}(S, M),
$$

where the group operation is given by $\left(\bar{f}_{1}, \mathcal{P}_{1}\right)\left(\bar{f}_{2}, \mathcal{P}_{2}\right)=\left(\bar{f}_{1} \bar{f}_{2}, \mathcal{P}_{1} \ominus f_{1}\left(\mathcal{P}_{2}\right)\right)$ for any $\mathcal{P}_{1}, \mathcal{P}_{2}$ in $\mathcal{Z}$ and any $f_{1}, f_{2}$ in $\operatorname{Homeo}^{+}(S, M)$. This group acts on the set of tagged triangulations of $(S, M)$, with the $\mathcal{Z}$ part acting by simultaneously changing the tags of the arcs attached to the punctures. 
On the one hand, since the orientation-preserving homeomorphism of $(S, M)$ and the changing of tags maintain the combinatorics of the tagged triangulations, an element in $\mathcal{M} \mathcal{G}_{\bowtie}(S, M)$ induces a direct cluster automorphism of the associating cluster algebra $\mathcal{A}(S, M)$ (see section 4.4 in [ASS12] for more details). On the other hand, a direct cluster automorphism in $A u t^{+}(\mathcal{A}(S, M))$ induces an isomorphism between the quivers of two tagged triangulations of $(S, M)$, and it is proved in [BS13] (Subsection 8.7) that except some special cases, the two tagged triangulations

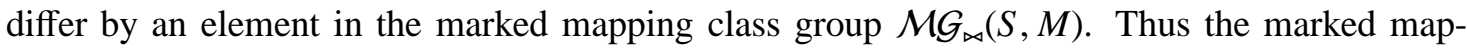
ping class group $\mathcal{M G _ { 凶 }}(S, M)$ and the direct automorphism group $A u t^{+}(\mathcal{A}(S, M))$ are isomorphic. More precisely, we have the following result:

Let $(S, M)$ be a surface satisfies the following

Assumption 1: Suppose that $(S, M)$ is not one of the following surfaces

(a) a once-punctured disc with two or four marked points on the boundary;

(b) a twice-punctured disc with two marked points on the boundary;

(c) a closed surface (which we have eliminated in the initial assumption of the surfaces).

Then the marked mapping class group $\mathcal{M G}_{\bowtie}(S, M)$ is isomorphic to the direct automorphism group $A u t^{+}(\mathcal{A}(S, M))$ (see also in [BQ12]).

\section{Cluster automorphism groups and coefficients}

We study in this section the influence of coefficients on cluster automorphism groups.

\subsection{Gluing free cluster algebras}

In this subsection, we fix $\left(Q, \mathbf{x}, \mathcal{A}_{Q}\right)$ a rooted cluster algebra with exchange matrix $B=\left(b_{j i}\right)_{(m+n) \times n}$.

Definition 3.1. We say that

(a) two frozen vertices $j$ and $k$ are glueable in $Q$, if there is a positive rational number $n_{j k}$ such that $b_{j i}=n_{j k} b_{k i}$ for any exchangeable vertex $i$ in $Q$;

(b) two frozen vertices $j$ and $k$ are strictly glueable in $Q$, if they are glueable with $n_{j k}=1$;

(c) a frozen vertex $j$ is prime, if g.c.d $\left(b_{j 1}, \cdots, b_{j n}\right)=1$;

(d) $Q$ is strictly gluing free, if any two frozen vertices in $Q$ are not glueable;

(e) $Q$ is gluing free, if any two frozen vertices in $Q$ are not strictly glueable;

(f) $Q$ is prime, if any frozen vertex is prime;

(g) $Q$ is prime gluing free, if $Q$ is prime and strictly gluing free;

(h) the rooted cluster algebra $\left(Q, \mathbf{x}, \mathcal{A}_{Q}\right)$ is gluing free, if $Q$ is gluing free;

(i) the rooted cluster algebra $\left(Q, \mathbf{x}, \mathcal{A}_{Q}\right)$ is prime gluing free, if $Q$ is prime gluing free. 
Remark 3.2. Note that $Q$ is prime gluing free if and only if it is prime and gluing free.

We separate the frozen vertices of $Q$ into sets of glueable vertices, then we have a disjoint collection of sets $\left\{j_{1,1}, \cdots, j_{1, t_{1}}\right\}, \cdots,\left\{j_{s, 1}, \cdots, j_{s, t_{s}}\right\}$ of frozen vertices of $Q$, where any two vertices (if there exist) in each set are glueable. Note that there may exist set with a single vertex. In fact $Q$ is strictly gluing free if and only if all of these sets have a single vertex. The vertex separation determines a separation of coefficients in $\mathcal{A}_{Q}$. We denote the corresponding sets by $\left\{x_{j_{1,1}}, \cdots, x_{j_{1, t_{1}}}\right\}, \cdots,\left\{x_{j_{s, 1}}, \cdots, x_{j_{s, t s}}\right\}$ respectively. Then we have the following

Theorem 3.3. For a rooted cluster algebra $\left(Q, \mathbf{x}, \mathcal{A}_{Q}\right)$, there is a unique prime gluing free rooted cluster algebra $\left(Q_{p g f}, \mathbf{x}_{p g f}, \mathcal{A}_{Q_{p g f}}\right)$ and a unique injective coefficient specialization $\varphi: \mathbb{P}_{p g f} \rightarrow \mathbb{P}$ such that for any $1 \leqslant k \leqslant s$, there is a coefficient $x_{j_{k}}$ in $\mathbb{P}_{p g f}$ which is mapped to $x_{j_{k, 1}}^{n_{j_{k, 1}}} x_{j_{k, 2}}^{n_{j_{k, 2}}} \cdots x_{j_{k, t_{k}}}^{n_{j_{k, t_{k}}}}$ in $\mathbb{P}$, where $n_{j_{k, 1}}, n_{j_{k, 2}}, \cdots, n_{j_{k, t_{k}}}$ are positive integer numbers, and $\mathbb{P}_{p g f}$ and $\mathbb{P}$ are coefficient groups of $\mathcal{A}_{Q_{p g f}}$ and $\mathcal{A}_{Q}$ respectively.

Proof. We prove this by several steps:

Step 1 . We construct the needed prime gluing free quiver $Q_{p g f}$ (write as $\bar{Q}$ for simplicity) from $Q$. The principal part of $\bar{Q}$ is the same as the principal part of $Q$. There are $s$ frozen vertices $\left\{j_{1}, \cdots, j_{s}\right\}$ in $\bar{Q}$, which is the set of representatives of the sets of glueable vertices in $Q$. We define the associating matrix $\bar{B}=\left(\bar{b}_{j i}\right)_{(n+s) \times n}$ of $\bar{Q}$ as follows. For each frozen vertex $j_{k}, 1 \leqslant k \leqslant s$, let $n_{j_{k, l}}$ in $\mathbb{Z}^{+}, 1 \leqslant l \leqslant s_{k}$, be the greatest common divisor of $\left\{b_{j_{k, l}}, 1 \leqslant i \leqslant n\right\}$. Then we have $\operatorname{g.c.d}\left(\frac{b_{j_{k, 1}}}{n_{j_{k, l}}}, \cdots, \frac{b_{j_{k, 1} n}}{n_{j_{k, l}}}\right)=1$. Let

$$
\bar{b}_{j_{k} i}=\frac{b_{j_{k, l} i}}{n_{j_{k, l}}}
$$

for any $1 \leqslant k \leqslant s$ and any $1 \leqslant i \leqslant n$. Note that $\bar{b}_{j_{k} i}$ is independent on the choice of $l$. In fact, all of the order sets $\left\{\frac{b_{j_{k, l}} 1}{n_{j_{k, l}}}, \cdots, \frac{b_{j_{k, 1}}}{n_{j_{k, l}}}\right\}, 1 \leqslant l \leqslant s_{k}$, are the same, since all of vertices $j_{k, l}, 1 \leqslant l \leqslant s_{k}$ are glueable with each other. Then by the construction, $\bar{Q}$ is prime gluing free.

Step 2. To show that $\varphi: \overline{\mathbb{P}} \rightarrow \mathbb{P}$ gives a coefficient specialization, by lemma 2.9. we only need to prove the equalities

$$
\varphi\left(\bar{p}_{i, \varpi}^{ \pm}\right)=p_{i, \varpi}^{ \pm}
$$

for any exchangeable vertex $i$ and admissible sequence $\varpi$.

For the case $\varpi=0, \bar{p}_{i}^{+}=\prod_{\bar{b}_{j_{k} i}>0} x_{j_{k}}^{\bar{b}_{j_{k} i}}$ and $p_{i}^{+}=\prod_{b_{j_{k, l} i}>0} x_{j_{k, l}}^{b_{j_{k, l}}}$, we have equalities:

$$
\begin{aligned}
& \varphi\left(\bar{p}_{i}^{+}\right)=\prod_{\bar{b}_{j_{k} i}>0} \varphi\left(x_{j_{k}}\right)^{\bar{b}_{j_{k} i}} \\
& =\prod_{\bar{b}_{j_{k} i}>0}\left(x_{j_{k, 1}}^{n_{j_{k, 1}}} \cdots x_{j_{k, t_{k}}}^{n_{j_{k} t_{k}}}\right)^{\bar{b}_{j_{k} i}} \\
& =\prod_{b_{j_{k} i}>0} x_{j_{k, 1}}^{b_{j_{k, 1} i}} \cdots x_{j_{k, t_{k}}}^{b_{j_{k, t_{k} i}}} \\
& =\prod_{b_{j_{k, l}}>0} x_{j_{k, l}}^{b_{k_{k}} i^{i}} \\
& =p_{i}^{+} \text {. }
\end{aligned}
$$


Similarly, we have $\varphi\left(\bar{p}_{i}\right)=p_{i}^{-}$.

For the case $\varpi=\left(i^{\prime}\right)$, where $i^{\prime}$ is an exchangeable vertex, we denote by $\left(Q^{\prime}, \mathbf{x}^{\prime}, \mathcal{A}_{Q^{\prime}}\right)$ and $\left(\bar{Q}^{\prime}, \overline{\mathbf{x}}^{\prime}, \mathcal{A}_{\bar{Q}^{\prime}}\right)$ the mutations $\left(\mu_{i^{\prime}}(Q), \mu_{i^{\prime}}(\mathbf{x}), \mathcal{A}_{\mu^{\prime}}(Q)\right)$ and $\left(\mu_{i^{\prime}}(\bar{Q}), \mu_{i^{\prime}}(\overline{\mathbf{x}}), \mathcal{A}_{\mu_{i^{\prime}}(\bar{Q})}\right)$ respectively. We denote by $B^{\prime}=\left(b_{j i}^{\prime}\right)_{(n+m) \times n}$ and $\bar{B}^{\prime}=\left(\bar{b}_{j i}^{\prime}\right)_{(n+s) \times n}$ the corresponding exchange matrices of $Q^{\prime}$ and $\bar{Q}^{\prime}$ respectively. Then it is clearly that the exchange part of $Q^{\prime}$ and $\bar{Q}^{\prime}$ are the same. For any frozen vertex $j_{k, l}$ in $Q$,

$$
b_{j_{k, l} i}^{\prime}= \begin{cases}b_{j_{k, l} i}+b_{j_{k, l^{\prime}}} b_{i^{\prime} i} & \text { if } i \neq i^{\prime} \text { and } b_{j_{k, l^{\prime}}} b_{i^{\prime} i} \geqslant 0 \\ b_{j_{k, l} i}-b_{j_{k, l} l^{\prime}} b_{i^{\prime} i} & \text { if } i \neq i^{\prime} \text { and } b_{j_{k, l} l^{\prime}} b_{i^{\prime} i}<0 \\ -b_{j_{k, l} i} & \text { if } i=i^{\prime}\end{cases}
$$

For any frozen vertex $j_{k}$ in $\bar{Q}$,

$$
\bar{b}_{j_{k} i}^{\prime}= \begin{cases}\bar{b}_{j_{k} i}+\bar{b}_{j_{k} i^{\prime}} \bar{b}_{i^{\prime} i} & \text { if } i \neq i^{\prime} \text { and } \bar{b}_{j_{k} i} \bar{b}_{i^{\prime} i} \geqslant 0 \\ \bar{b}_{j_{k} i}-\bar{b}_{j_{k} i^{\prime}} \bar{b}_{i^{\prime} i} i & \text { if } i \neq i^{\prime} \text { and } \bar{b}_{j_{k} i} \bar{b}_{i^{\prime} i}<0 \\ -\bar{b}_{j_{k} i} & \text { if } i=i^{\prime}\end{cases}
$$

Note that $n_{j_{k, l}}>0$, then from $b_{j_{k, l} i}=n_{j_{k, l}} \bar{b}_{j_{k} i}, b_{j_{k, l} l^{\prime}}=n_{j_{k, l}} \bar{b}_{j_{k} i^{\prime}}$ and $b_{i^{\prime} i}=\bar{b}_{i^{\prime} i}$, we have $b_{j_{k, l}}^{\prime}=$ $n_{j_{k, l}} \bar{b}_{j_{k} i}^{\prime}$. Let $g=\operatorname{g.c.d}\left(\bar{b}_{j_{k} 1}^{\prime}, \cdots, \bar{b}_{j_{k} n}^{\prime}\right)$, then $g \mid \bar{b}_{j_{k} i^{\prime}}^{\prime}=-\bar{b}_{j_{k} i^{\prime}}$, and thus $g \mid \bar{b}_{j_{k} i}$ for any $1 \leqslant i \leqslant n$. Because g.c.d $\left(\bar{b}_{j_{k} 1}, \cdots, \bar{b}_{j_{k} n}\right)=1$, we have $g=1$. Thus $\operatorname{g.c} . d\left(b_{j_{k, l}}^{\prime}, \cdots, b_{j_{k, l}}^{\prime}\right)=n_{j_{k, l},}$. Therefore from the construction of the prime gluing free quiver, we have $\left(\mu_{i^{\prime}}(Q)\right)_{p g f}=\mu_{i^{\prime}}\left(Q_{p g f}\right)$. Then by the case $\varpi=0, \varphi\left(\bar{p}_{i,\left(i^{\prime}\right)}^{ \pm}\right)=p_{i,\left(i^{\prime}\right)}^{ \pm}$. Finally, we prove the equalities 3 by inductions.

Step 3. Since $\mathbb{P}_{p g f}$ and $\mathbb{P}$ are both free abelian groups, from the construction of the coefficient specialization $\varphi$, it is not hard to see that $\varphi$ is injective. The injectivity of $\varphi$ yields that there are no more coefficients in $\mathcal{A}_{Q_{p g} f}$, excepting the ones $j_{1}, \cdots, j_{s}$, this guarantees the uniqueness of $\left(Q_{p g f}, \mathbf{x}_{p g f}, \mathcal{A}_{Q_{p g f}}\right)$.

Remark 3.4. (a) It is not hard to see that $\varphi$ not only maps $\mathcal{A}_{Q_{p g f}}\left[\mathbb{P}_{p g f}^{-1}\right]$ to $\mathcal{A}_{Q}\left[\mathbb{P}^{-1}\right]$, but also induces an injective algebra homomorphism from $\mathcal{A}_{Q_{p g f}}$ to $\mathcal{A}_{Q}$, which maps exchangeable cluster variables to exchangeable ones. We call $Q_{p g f}$ the prime gluing free quiver of $Q$ and $\left(Q_{p g f}, \mathbf{x}_{p g f}, \mathcal{A}_{Q_{p g f}}\right)$ the prime gluing free rooted cluster algebra of $\left(Q, \mathbf{x}, \mathcal{A}_{Q}\right)$.

(b) We consider the disjoint collection of sets $\left\{j_{1,1}, \cdots, j_{1, t_{1}}\right\}, \cdots,\left\{j_{s, 1}, \cdots, j_{s, t_{s}}\right\}$ of frozen vertices of $Q$, where any two vertices (if there exist) in each set are strictly glueable. Then we define $Q_{g f}$ as the quiver obtained from $Q$ by deleting the vertices $j_{k, l}$ for any $1 \leqslant k \leqslant s$ and $2 \leqslant l \leqslant s_{k}$. We denote by $j_{k}=j_{k, 1}$ for simplicity. Then $Q_{g f}$ is gluing free. We call it the gluing free quiver of $Q$. It is not hard to see that we have similar results as in above theorem. That is, there is a unique injective coefficient specialization $\varphi: \mathbb{P}_{g f} \rightarrow \mathbb{P}$ such that for any $1 \leqslant k \leqslant s, \varphi\left(x_{j_{k}}\right)=x_{j_{k, 1}} x_{j_{k, 2}} \cdots x_{j_{k, t_{k}}}$. Similarly, $\varphi$ induces an injective algebra homomorphism from $\mathcal{A}_{Q_{g f}}$ to $\mathcal{A}_{Q}$, which maps exchangeable cluster variables to exchangeable ones. We call $\left(Q_{g f}, \mathbf{x}_{g f}, \mathcal{A}_{Q_{g f}}\right)$ the gluing free rooted cluster algebra of $\left(Q, \mathbf{x}, \mathcal{A}_{Q}\right)$.

Definition 3.5. Let $\left(Q^{\prime}, \mathbf{x}^{\prime}, \mathcal{A}_{Q^{\prime}}\right)$ and $\left(Q^{\prime \prime}, \mathbf{x}^{\prime \prime}, \mathcal{A}_{Q^{\prime \prime}}\right)$ be two rooted cluster algebras. We say that $Q^{\prime}\left(\left(Q^{\prime}, \mathbf{x}^{\prime}, \mathcal{A}_{Q^{\prime}}\right)\right.$ respectively) and $Q^{\prime \prime}\left(\left(Q^{\prime \prime}, \mathbf{x}^{\prime \prime}, \mathcal{A}_{Q^{\prime \prime}}\right)\right.$ respectively) are gluing equivalent, if their prime gluing free quivers are isomorphic. 
Note that the gluing equivalent relation gives rise to an equivalent relation on ice quivers (thus on rooted cluster algebras). By this equivalent relation, we separate ice quivers (rooted cluster algebras respectively) to gluing equivalent classes, which consist of the gluing equivalent ice quivers (gluing equivalent rooted cluster algebras respectively). Each equivalent class is uniquely determined by a prime gluing free ice quiver. This prime gluing free ice quiver is a universal element in the sense of that there is a coefficient specialization from this quiver to any quiver in the equivalent class.

Lemma 3.6. Let $Q$ be an ice quiver and $\bar{Q}$ be its prime gluing free quiver (or gluing free quiver).

(a) For any admissible sequence $\varpi, \mu_{\varpi}(\bar{Q})$ is the prime gluing free quiver (or gluing free quiver respectively) of $\mu_{\varpi}(Q)$;

(b) For any admissible sequence $\varpi, \mu_{\varpi}(\bar{Q}) \cong \bar{Q}\left(\right.$ or $\left.\bar{Q}^{\text {op }}\right)$ if and only if $\mu_{\varpi}(Q) \cong Q\left(\right.$ or $\left.Q^{o p}\right)$.

Proof. Part (a) follows from Step 2 in the proof of above theorem that the construction of a prime gluing free quiver (or gluing free quiver) commutates with the quiver mutation. Part $(b)$ follows from $(a)$ and the uniqueness of a prime gluing free quiver (or gluing free quiver respectively).

Definition 3.7. We say a cluster algebra $\mathcal{A}$ prime gluing free (gluing free respectively), if there is a rooted cluster algebra $(Q, \mathbf{x}, \mathcal{A})$ of $\mathcal{A}$ such that $Q$ is prime gluing free ( gluing free respectively).

Then it follows from Lemma 3.6 that a cluster algebra is prime gluing free (gluing free respectively), if and only if all of its exchange quivers are prime gluing free (gluing free respectively). We define $\mathcal{A}_{\bar{Q}}$ constructed in Theorem 3.3 (Remark 3.4 b) respectively) as the prime gluing free (gluing free respectively) cluster algebra of $\mathcal{A}_{Q}$.

Let $B_{n \times n}$ be an skew-symmetric square matrix, we call a cluster algebra $\mathcal{A}^{p r}$ with the initial matrix $B^{p r}=\left(\begin{array}{l}B \\ I_{n}\end{array}\right)$ a cluster algebra with principal coefficients, where $I_{n}$ is an identity matrix.

Proposition 3.8. Any cluster algebra with principal coefficients is prime gluing free.

Proof. This is clearly.

Proposition 3.9. Any universal geometric cluster algebra is prime gluing free.

Proof. Let $\left(Q, \mathbf{x}, \mathcal{A}_{Q}\right)$ be a rooted cluster algebra such that $\mathcal{A}_{Q}$ is a universal geometric cluster algebra with coefficient group $\mathbb{P}$. Denote by $B=\left(b_{j i}\right)_{(m+n) \times n}$ the matrix associated to $Q$. To show that $\mathcal{A}_{Q}$ is prime gluing free, it is sufficient to show that $Q$ is prime gluing free.

Firstly, we show that all the vertices in $Q$ are prime. Suppose that a frozen vertex $j_{1}$ of $Q$ is not prime. Denote by $q=$ g.c.d $\left(b_{j_{1} 1}, \cdots, b_{j_{1} n}\right) \in \mathbb{Z}^{+}$the greatest common divisor of the integers in $j_{1}$ row of $B$. Let $\bar{B}$ be the matrix obtained from $B$ by replacing the elements $b_{j_{1} i}$ by $\frac{b_{j_{1} i}}{q}$ for any $1 \leqslant i \leqslant n$. Denote by $\bar{Q}$ the ice quiver associated to $\bar{B}$. Then we have a rooted cluster algebra $(\bar{Q}, \overline{\mathbf{x}}, \mathcal{A} \bar{Q})$ in $R C A\left(Q^{e x}\right)$, and we denote its coefficient group by $\overline{\mathbb{P}}$. Define a group homomorphism $\varphi: \overline{\mathbb{P}} \rightarrow \mathbb{P}$ such that $\varphi\left(\bar{x}_{j_{1}}\right)=x_{j_{1}}{ }^{q}$ and $\varphi\left(\bar{x}_{j}\right)=x_{j}$ for the other frozen variables $\bar{x}_{j}$ in $\overline{\mathbf{x}}$. Then it is not hard to see that $\varphi$ is a coefficient specialization. On the one hand, since a composition of any two coefficient specialization is also a coefficient specialization, $\mathcal{A}_{\bar{Q}}$ is a universal geometric 
cluster algebra with principal part $Q^{e x}$. By the uniqueness of the coefficient specialization, $\varphi$ is the only coefficient specialization from $\overline{\mathbb{P}}$ to $\mathbb{P}$, but note that the matrix of $\varphi$ is not invertible. This contradicts the uniqueness of the universal geometric cluster algebra.

Secondly, we show that $Q$ is strictly gluing free. Suppose that $Q$ is not strictly gluing free, since $Q$ is prime, this means that there exist two strictly glueable frozen vertices $j_{1}$ and $j_{2}$ in $Q$. We define $\varphi$ as a map from $\mathbb{P}$ to $\mathbb{P}$ with $\varphi\left(x_{j_{1}}\right)=x_{j_{2}}, \varphi\left(x_{j_{2}}\right)=x_{j_{1}}$ and $\varphi\left(x_{j_{k}}\right)=x_{j_{k}}$ for the other frozen variables in $\mathbf{x}$. Then $\varphi$ induces a group homomorphism from $\mathbb{P}$ to $\mathbb{P}$ and in fact a coefficient specialization, which is different from the identity coefficient specialization. This contradicts the uniqueness of the coefficient specialization. Thus $Q$ is gluing free, and we are done.

Proposition 3.10. Let $(S, M)$ be an oriented marked Riemann surface. Assume that $(S, M)$ is not a 4-gon. Then the cluster algebras $\mathcal{A}^{b}(S, M)$ associated to $(S, M)$ with coefficients is prime gluing free.

Proof. To prove that $\mathcal{A}^{b}(S, M)$ is prime gluing free, we only need to find a prime gluing free quiver of $\mathcal{A}^{b}(S, M)$. Assume that $T$ is an ideal triangulation without self-floded triangles, Lemma 2.11 makes sense the assumption. Let $T^{b}$ be the corresponding triangulation with boundary. Denote by $Q^{b}$ the quiver of $T^{b}$. We unify the $\operatorname{arcs}$ in $T^{b}$ with the vertices in $Q^{b}$. Firstly, since each boundary is contained in a single triangle, there is at most one arrow between its corresponding vertex and any exchangeable vertex. Thus in $Q^{b}$, each frozen vertex is prime. Secondly, we prove that $\mathcal{A}^{b}(S, M)$ is gluing free. Since there are no self-floded triangles in $T$, the type I triangle in Figure 11 is the only possible puzzle piece of $T^{b}$. Let $j$ be a frozen vertex in $Q^{b}$, assume that $j$ is adjacent to an exchangeable vertex $i$. Without loss of generality, we assume that they are in the following triangles, where the orientation is clockwise. Then there is an arrow from $j$ to $i$. If there exists a frozen vertex $k$ glueable with $j$, then by the orientation of the surface, it must be in the following position. We claim that the vertex $a c$ must be frozen. In fact, if $a c$ is exchangeable, then there is an arrow from $a c$ to $j$. Since $j$ and $k$ are glueable, there is an arrow from $a c$ to $k$. Thus $a c$ and $k$ must be in a triangle of $T^{b}$, which is depicted as $\Delta a c d$ in the picture. Therefore $k$ is an exchangeable vertex, and we have a contradiction. Similarly, $b d$ is also a frozen vertex. Thus $(S, M)$ is a 4-gon, a contradiction to the assumption. Therefore $\mathcal{A}^{b}(S, M)$ is gluing free. We are done.

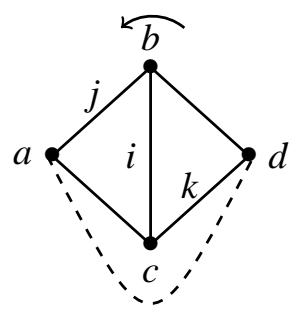

Example 2. We consider the 4-gon with the following triangulation. 

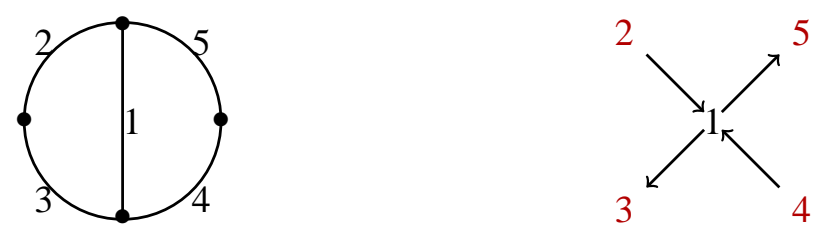

Then in its ice quiver $Q^{b}$, the frozen vertices 2 and 4,3 and 5 are strictly glueable. Therefore $Q^{b}$ is not gluing free.

Theorem 3.11. Let $\left(Q, \mathbf{x}, \mathcal{A}_{Q}\right)$ be a rooted cluster algebra and $\left(\bar{Q}, \overline{\mathbf{x}}, \mathcal{A}_{\bar{Q}}\right)$ be its gluing free rooted cluster algebra. Let $\left\{j_{1,1}, \cdots, j_{1, t_{1}}\right\}, \cdots,\left\{j_{s, 1}, \cdots, j_{s, t_{s}}\right\}$ be the collections of strictly glueable vertices of $Q$. Then

(a) there is an injective group homomorphism $\Phi$ from Aut $\left(\mathcal{A}_{Q}\right)$ to $\operatorname{Aut}\left(\mathcal{A}_{\bar{Q}}\right) \times S_{t_{1}} \times \cdots \times S_{t_{s}}$;

(b) there is an injective group homomorphism $\Phi^{+}$from $A u t^{+}\left(\mathcal{A}_{Q}\right)$ to $A u t^{+}\left(\mathcal{A}_{\bar{Q}}\right) \times S_{t_{1}} \times \cdots \times S_{t_{s}}$.

Proof. We use the notations in the Remark 3.4(b). Let $\phi: \mathcal{A}_{Q} \rightarrow \mathcal{A}_{Q}$ be a cluster automorphism of $\mathcal{A}_{Q}$. Then it induces a bijection from $\mathbf{x}$ to $\mu_{\varpi}(\mathbf{x})$ for some admissible sequence $\varpi$, and a permutation $\sigma$ on the vertex set $Q_{0}$ of $Q$ such that $\phi\left(x_{i}\right)=\mu_{\varpi}\left(x_{\sigma(i)}\right)$ for any $x_{i} \in \mathbf{x}$. And $\sigma$ induces a permutation $\sigma^{\prime}$ on the frozen vertices of $Q$. Since $\phi$ is an automorphism, $\sigma^{\prime}$ maps strictly glueable vertices to the strictly glueable ones. Thus $\sigma^{\prime}$ induces a permutation on the partition $\left\{j_{1,1}, \cdots, j_{1, t_{1}}\right\}, \cdots,\left\{j_{s, 1}, \cdots, j_{s, t_{s}}\right\}$. Therefore $\sigma^{\prime}$ induces a permutation $\sigma^{\prime \prime}$ on the frozen vertices $\left\{j_{1}, \cdots, j_{s}\right\}$ of $\bar{Q}$ which maps $j_{k}$ to $j_{\sigma^{\prime \prime}(k)}$ for each $1 \leqslant k \leqslant s$. Then we can write $\sigma^{\prime}$ as $\left(\sigma^{\prime \prime}, \sigma_{1}, \cdots, \sigma_{s}\right)$ by mapping each $j_{k, l}$ to $j_{\sigma^{\prime \prime}(k), \sigma_{k}(l)}$, where $\sigma_{k}$ is a permutation on $\left\{1, \cdots, t_{k}\right\}$. Define $\phi^{\prime}: \overline{\mathbf{x}} \rightarrow \mu_{\varpi}(\overline{\mathbf{x}})$ as a map by $\phi^{\prime}\left(\bar{x}_{i}\right)=\mu_{\varpi}\left(\bar{x}_{\sigma(i)}\right)$ for any exchangeable vertex $i$ of $\bar{Q}$ and $\phi^{\prime}\left(\bar{x}_{j_{k}}\right)=\mu_{\varpi}\left(\bar{x}_{j_{\sigma^{\prime \prime}(k)}}\right)$ for any $1 \leqslant k \leqslant s$. Since $\varphi$ is a cluster automorphism of $\mathcal{A}_{Q}$, by Proposition 2.5 (a), it maps $Q$ to an ice quiver which is isomorphic to itself or to an opposite quiver. Therefore by using Proposition 2.5 (a) again, Lemma 3.6 (b) shows that $\phi^{\prime}$ induces a cluster automorphism $\phi^{\prime \prime}$ of $\mathcal{A}_{\bar{Q}}$. We define $\left(\phi^{\prime \prime}, \sigma_{1}, \cdots, \sigma_{s}\right)$ as an element $\operatorname{Aut}\left(\mathcal{A}_{\bar{Q}}\right) \times S_{t_{1}} \times \cdots \times S_{t_{s}}$. Then it is not hard to see that $\Phi: \phi \rightarrow\left(\phi^{\prime \prime}, \sigma_{1}, \cdots, \sigma_{s}\right)$ is an injective group homomorphism. The second group homomorphism follows from the first one and Lemma 3.6 (b).

Remark 3.12. Note that a permutation of strictly glueable vertices of $Q$ induces an automorphism of $Q$, then it induces an automorphism of $\mathcal{A}_{Q}$. Thus $\Phi$ is surjective onto $S_{t_{1}} \times \cdots \times S_{t_{s}}$. However, $\Phi$ may not be a surjection, see in the following example. We consider the following ice quiver $Q$ with frozen vertices 3, 4 and 5 . Then its gluing free quiver is $\bar{Q}$, where 3 and 4 are frozen vertices.<smiles>O=[Te](S)([Hg])[Hg]</smiles>$$
\bar{Q}: \quad 3 \longrightarrow 1 \longrightarrow 2 \longleftarrow 4
$$

Then $\mu_{1} \mu_{2}(\bar{Q}) \cong \bar{Q}^{\text {op }}$, and it induces a opposite cluster automorphism $\phi$ of $\mathcal{A}_{\bar{Q}}$. However $\mu_{1} \mu_{2}(Q)$ is neither isomorphic to $Q$ nor to $Q^{o p}$. Thus $\phi$ is not an image of $\Phi$. 


\subsection{Exchange graphs}

Recall from [FZ02] and [FZ07] that for a cluster algebra $\mathcal{A}_{Q}$, where $Q$ is an ice quiver with $n$ exchangeable vertices, the exchange graph $E_{Q}$ of $\mathcal{A}_{Q}$ is a $n$-regular graph whose vertices are the seeds of $\mathcal{A}_{Q}$ and two vertices are connected by an edge labeled by $x_{i}, 1 \leqslant i \leqslant n$, if and only if the corresponding seeds are adjacent in direction $x_{i}$. In our settings, the clusters determine the quivers, thus the vertices of $E_{Q}$ are in fact the clusters of $\mathcal{A}_{Q}$. It is proved in [CKLP13](Theorem 4.6) that $E_{Q}$ only depends on the principal part $Q^{e x}$, that is, there is an isomorphism from $E_{Q}$ to $E_{Q^{e x}}$. The correspondence on the vertices is given by the specialization $S_{E}$ : for each vertex $\mathbf{x}$ on $E_{Q}, S_{E}(\mathbf{x})$ is obtained from $\mathbf{x}$ by specializing any frozen variable in the exchangeable variables to integer 1, and forgetting the frozen part of $\mathbf{x}$.

Now we introduce the following notion:

Definition 3.13. An automorphism of $E_{Q}$ is an automorphism of $E_{Q}$ as a graph, that is, a permutation $\sigma$ of the vertex set, such that the pair of vertices $(u, v)$ forms an edge if and only if the pair $(\sigma(u), \sigma(v))$ also forms an edge.

Then an automorphism of $E_{Q}$ gives a bijection on the vertices and on the edges of $E_{Q}$, and maps adjacent vertices to adjacent ones. It is clearly that the natural composition of two automorphisms of $E_{Q}$ is again an automorphism of $E_{Q}$. We define an automorphism group $\operatorname{Aut}\left(E_{Q}\right)$ of $E_{Q}$ as a group consisting of automorphisms of $E_{Q}$ with compositions of automorphisms as multiplications. Then the isomorphism $S_{E}: E_{Q} \rightarrow E_{Q^{e x}}$ induces a group isomorphism from $\operatorname{Aut}\left(E_{Q}\right)$ to $\operatorname{Aut}\left(E_{Q^{e x}}\right)$, we also denote it by $S_{E}$.

Example 3. We consider the cluster algebra of type $A_{3}$ with initial seed $\left(Q,\left\{x_{1}, x_{2}, x_{3}\right\}\right)$, where $Q$ is $1 \longrightarrow 2 \longrightarrow 3$. Then its exchange graph $E_{Q}$ is depicted in Figure 2 where the vertex $O$ represents the seed $\left(Q,\left\{x_{1}, x_{2}, x_{3}\right\}\right)$. Note that there are three quadrilaterals and six pentagons in $E_{Q}$. Since an automorphism of a graph maps vertices to vertices, edges to edges and thus faces to faces, it is not hard to see that the automorphism group Aut $\left(E_{Q}\right)$ is generated by $\tau$ and $\sigma$ showed in Figure 2 The automorphism $\tau$ is given by the dashed lines, maps red edges to red ones and maps green edges to green ones. The automorphism $\sigma$ is the reflection along the central axis. Note that the order of $\tau$ is 6 , the order of $\sigma$ is 2 and the only relation which they satisfy is $\sigma \tau=\tau^{5} \sigma$. Thus Aut $\left(E_{Q}\right)$ is isomorphic to the dihedral group $D_{6}$.

Theorem 3.14. Let $Q$ be an ice quiver with at least two exchangeable vertices.

(a) If $Q$ is gluing free, then the cluster automorphism group $A u t\left(\mathcal{A}_{Q}\right)$ is a subgroup of $\operatorname{Aut}\left(E_{Q}\right)$.

(b) If $Q$ is not gluing free, denoted by $\left\{j_{1,1}, \cdots, j_{1, t_{1}}\right\}, \cdots,\left\{j_{s, 1}, \cdots, j_{s, t_{s}}\right\}$ the collections of strictly glueable vertices of $Q$, then the cluster automorphism group Aut $\left(\mathcal{A}_{Q}\right)$ is a subgroup of $\operatorname{Aut}\left(E_{Q}\right) \times S_{t_{1}} \times \cdots \times S_{t_{s}}$.

Proof. (a) Let $f$ be an automorphism of $\mathcal{A}_{Q}$, then Proposition 2.5 (3) yields that $f$ gives rise to a map $f^{\prime}$ from the vertices of $E_{Q}$ to the vertices of $E_{Q}$. In fact $f^{\prime}$ is a bijection since $f$ is an automorphism. For any vertex $\mathbf{x}$ and its adjacent vertex $\mathbf{x}^{\prime}=\mathbf{x} \backslash\{x\} \sqcup\left\{\mu_{x}(x)\right\}$ in $E_{Q}$, we have $f\left(\mu_{x}(x)\right)=$ $\mu_{f(x)}(f(x))$ since $f$ is a cluster automorphism. Thus we have $f^{\prime}\left(\mathbf{x}^{\prime}\right)=f^{\prime}(\mathbf{x}) \backslash\{f(x)\} \sqcup\left\{\mu_{f(x)}(f(x)\}\right.$. Therefore the vertices $f^{\prime}(\mathbf{x})$ and $f^{\prime}\left(\mathbf{x}^{\prime}\right)$ are adjacent in $E_{Q}$. We have proved that $f^{\prime}$ is an automorphism of $E_{Q}$, and it is not hard to see that this yields a group homomorphism from $\operatorname{Aut}\left(\mathcal{A}_{Q}\right)$ 


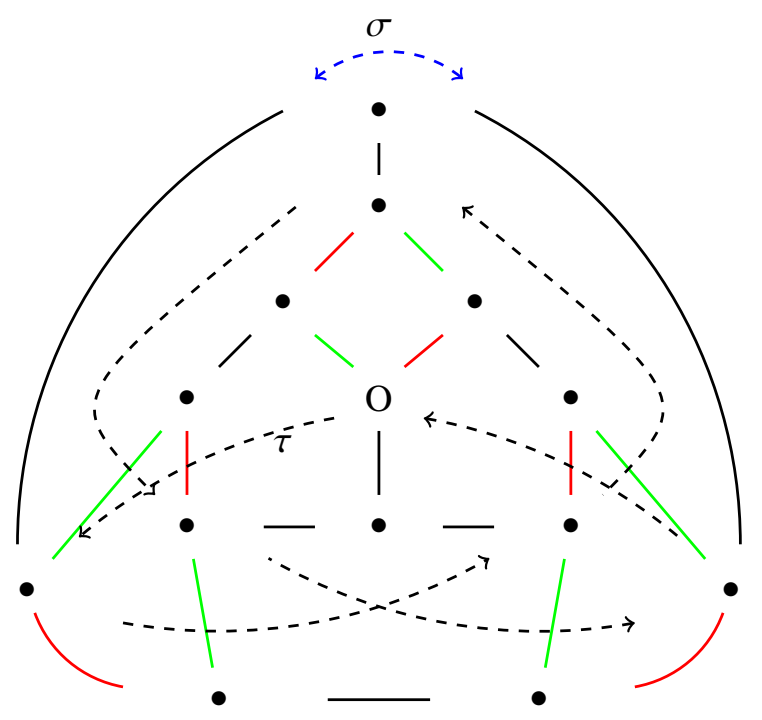

Figure 2: The automorphisms of exchange graph of type $A_{3}$

to $\operatorname{Aut}\left(E_{Q}\right)$. For the injectivity, if $f^{\prime}$ is an identity on $E_{Q}$, then $f$ fix any cluster, and it is not hard to see that it fix any exchangeable variable. On the other hand, since $Q$ is gluing free and $Q$ has at least two exchangeable vertices, any permutation of frozen variables does not induce an automorphism of $\mathcal{A}_{Q}$. Thus $f$ fixes any cluster variables, and it is an identity on $\mathcal{A}_{Q}$. Therefore $\operatorname{Aut}\left(\mathcal{A}_{Q}\right)$ is a subgroup of $\operatorname{Aut}\left(E_{Q}\right)$.

(b) Let $\bar{Q}$ be the gluing free quiver of $Q$, then it is gluing free. Therefore, by (a), $\operatorname{Aut}\left(\mathcal{A}_{\bar{Q}}\right)$ is a subgroup of $\operatorname{Aut}\left(E_{\bar{Q}}\right)$. Moreover, since $\operatorname{Aut}\left(E_{Q}\right) \cong \operatorname{Aut}\left(E_{\bar{Q}}\right)$ and $\operatorname{Aut}\left(\mathcal{A}_{Q}\right)$ is a subgroup of $\operatorname{Aut}\left(\mathcal{A}_{\bar{Q}}\right) \times S_{t_{1}} \times \cdots \times S_{t_{s}}$ by Theorem 3.11 $\operatorname{Aut}\left(\mathcal{A}_{Q}\right)$ is a subgroup of $\operatorname{Aut}\left(E_{Q}\right) \times S_{t_{1}} \times \cdots \times S_{t_{s}}$.

Example 4. If $Q$ is an ice quiver with a single exchangeable vertex, the results in Theorem 3.14 may be not true. We consider the cluster algebra $\mathcal{A}_{Q^{b}}$ of a 4-gon with the initial triangulation given in Example 2 Then the exchange graph of $\mathcal{A}_{Q^{b}}$ is a line segment with two vertices and its automorphism group Aut $\left(E_{Q^{b}}\right)$ is isomorphic to $S_{2}$.

On the other hand, Aut $\left(\mathcal{A}_{Q^{b}}\right) \cong<\sigma_{x_{1} \mu_{1}\left(x_{1}\right)}>\times<\sigma_{x_{2} x_{4}}>\times<\sigma_{x_{3} x_{5}}>\times<\sigma_{x_{2} x_{5}} \sigma_{x_{3} x_{4}}>\cong$ $S_{2} \times S_{2} \times S_{2} \times S_{2}$, where we denote by $\sigma_{x_{i} x_{j}}\left(\sigma_{x_{1} \mu_{1}\left(x_{1}\right)}\right.$ respectively) the automorphism of $\mathcal{A}_{Q^{b}}$ induced by the permutation of cluster variables $x_{i}$ and $x_{j}\left(x_{1}\right.$ and $\mu_{1}\left(x_{1}\right)$ respectively). Then $\operatorname{Aut}\left(\mathcal{A}_{Q^{b}}\right) \cong S_{2} \times S_{2} \times S_{2} \times S_{2}$ is not a subgroup of Aut $\left(E_{Q^{b}}\right) \times S_{2} \times S_{2} \cong S_{2} \times S_{2} \times S_{2}$.

Example 5. We consider the cluster algebra of type $A_{3}$ with initial seed $\left(Q,\left\{x_{1}, x_{2}, x_{3}\right\}\right)$, where $Q$ is $1 \longrightarrow 2 \longrightarrow 3$. As showed in Example 3 Aut $\left(E_{Q}\right)$ is isomorphic to the dihedral group $D_{6}$ generated by $\tau$ and $\sigma$. Here $\tau$ induces a bijection on the cluster variables of $\mathcal{A}_{Q}$, and we also 
denote it by $\tau$. In fact $\tau$ is a cluster automorphism which is induced by the AR-translation on the cluster category of the algebra $k Q$ (see Section 3 of [ASS12] for more details), it is given by:

$$
\tau:\left\{\begin{array}{rll}
x_{1} & \mapsto \frac{1+x_{2}}{x_{1}} \\
x_{2} & \mapsto \frac{x_{1}+x_{3}+x_{2} x_{3}}{x_{1} x_{2}} \\
x_{3} & \mapsto \frac{\left(1+x_{1}\right)\left(x_{1}+x_{3}\right)}{x_{1} x_{2} x_{3}} .
\end{array}\right.
$$

On the other hand, the automorphism $\sigma$ of $E_{Q}$ corresponds to the opposite automorphism of $\mathcal{A}_{Q}$ given by exchanging the cluster variables $x_{1}$ and $x_{2}$. Therefore $\operatorname{Aut}\left(E_{Q}\right) \subseteq \operatorname{Aut}\left(\mathcal{A}_{Q}\right)$. Note that from Theorem 3.14 $\operatorname{Aut}\left(\mathcal{A}_{Q}\right)$ is a subgroup of $\operatorname{Aut}\left(E_{Q}\right)$, thus $\operatorname{Aut}\left(\mathcal{A}_{Q}\right) \cong \operatorname{Aut}\left(E_{Q}\right) \cong D_{6}$.

Remark 3.15. In the subsequent paper $[C Z 15 b]$ we prove that for a coefficient free cluster algebra $\mathcal{A}_{Q}$, the two groups $A u t\left(\mathcal{A}_{Q}\right)$ and $A u t\left(E_{Q}\right)$ are isomorphic with each other, if $\mathcal{A}$ is of finite type, excepting types of rank two and type $F_{4}$, or $\mathcal{A}$ is of skew-symmetric finite mutation type. For a cluster algebra of type $F_{4}$ and all the non-skew-symmetric cluster algebra of rank two, $\operatorname{Aut}\left(\mathcal{A}_{Q}\right)$ is a proper subgroup of $\operatorname{Aut}\left(E_{Q}\right)$.

Example 6. Let $Q^{\prime}$ be the following quiver, where vertices 4 and 5 are frozen.

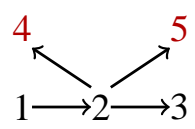

The seed $\left(Q^{\prime},\left\{x_{1}, x_{2}, x_{3}, x_{4}, x_{5}\right\}\right)$ defines a cluster algebra $\mathcal{A}_{Q^{\prime}}$. We consider the cluster automorphism group Aut $\left(\mathcal{A}_{Q^{\prime}}\right)$. Note that the vertices 4 and 5 are strictly glueable, thus exchanging the corresponding cluster variables $x_{4}$ and $x_{5}$ induces a cluster automorphism $\delta$ of Aut $\left(\mathcal{A}_{Q^{\prime}}\right)$, whose order is two, thus $\{1, \delta\} \cong S_{2}$ is a subgroup of $A u t\left(\mathcal{A}_{Q^{\prime}}\right)$. On the other hand, we consider the gluing free quiver of $Q^{\prime}$ :

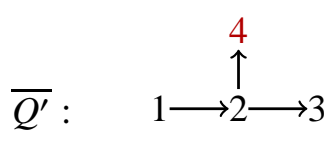

The exchange graph of $\mathcal{A}_{\overline{Q^{\prime}}}$ is depicted in Figure 3 which is isomorphic to the one in Figure 2 An automorphism in Aut $\left(\mathcal{A}_{\overline{Q^{\prime}}}\right)$ maps $\overline{Q^{\prime}}$ to a quiver isomorphic to $\overline{Q^{\prime}}$ or to ${\overline{Q^{\prime}}}^{\text {op }}$. A direct compute shows that there are two seeds satisfy the above property: $\mu_{1} \mu_{3}\left(\overline{Q^{\prime}}\right) \cong \overline{Q^{\prime}}, \mu_{3} \mu_{2} \mu_{1} \mu_{2}\left(\overline{Q^{\prime}}\right) \cong{\overline{Q^{\prime}}}^{o p}$. Then the automorphisms of $\mathcal{A}_{\overline{Q^{\prime}}}$ is showed in Figure 3 (compare with Figure 2), where $\tau$ and $\sigma$ are algebra homomorphisms determined by:

$$
\tau:\left\{\begin{array}{rll}
x_{1} & \mapsto \frac{1+x_{2}}{x_{1}} \\
x_{2} & \mapsto \frac{x_{1}+x_{3} x_{4}+x_{2} x_{3} x_{4}}{x_{1} x_{2}} \\
x_{3} & \mapsto \frac{\left(1+x_{1}\right)\left(x_{1}+x_{3} x_{4}\right)}{x_{1} x_{2} x_{3}} \\
x_{4} & \mapsto x_{4}
\end{array}\right.
$$

and

$$
\sigma:\left\{\begin{array}{rll}
x_{1} & \mapsto & x_{3} \\
x_{2} & \mapsto & x_{2} \\
x_{3} & \mapsto & x_{1} \\
x_{4} & \mapsto & x_{4} .
\end{array}\right.
$$

Then $\operatorname{Aut}\left(\mathcal{A}{\overline{Q^{\prime}}}\right)=\left\{1, \tau^{3}, \tau \sigma, \tau^{4} \sigma\right\} \cong K_{4}$ is a proper subgroup of Aut $\left(E_{\bar{Q}}\right)$, which is isomorphic to $D_{6}$. Finally we have $\operatorname{Aut}\left(\mathcal{A}_{Q^{\prime}}\right) \cong \operatorname{Aut}\left(\mathcal{A}_{\overline{Q^{\prime}}}\right) \times\{1, \delta\} \cong S_{2} \times S_{2} \times S_{2}$. 


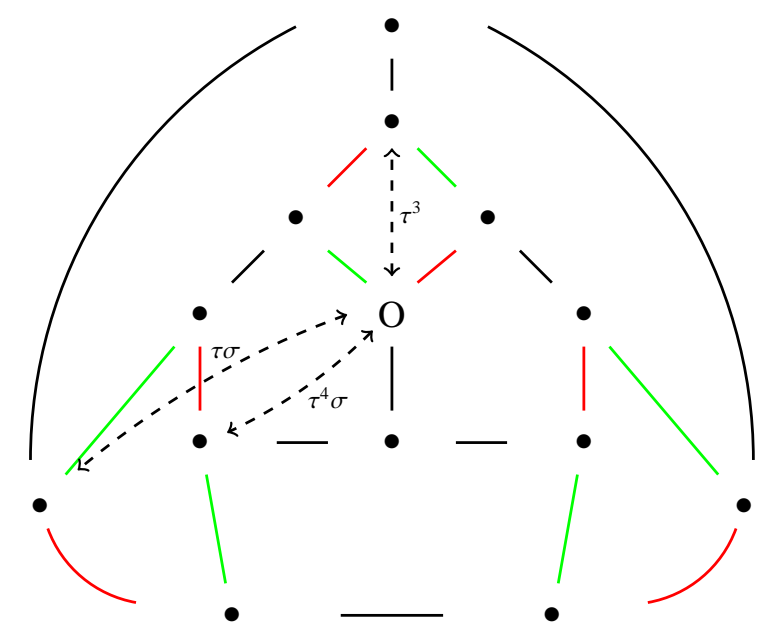

Figure 3: The automorphisms of a cluster algebra of type $A_{3}$ with a coefficient

\subsection{Main results}

Theorem 3.16. Let $Q^{\prime}$ be an ice quiver with principal part $Q$. Assume that there are at least two vertices in $Q$.

(a) If $Q^{\prime}$ is gluing free, then $\operatorname{Aut}\left(\mathcal{A}_{Q^{\prime}}\right)$ is a subgroup of $\operatorname{Aut}\left(\mathcal{A}_{Q}\right)$, and $A u t^{+}\left(\mathcal{A}_{Q^{\prime}}\right)$ is a subgroup of $\operatorname{Aut}^{+}\left(\mathcal{A}_{Q}\right)$.

(b) If $Q^{\prime}$ is not gluing free, denoted by $\left\{j_{1,1}, \cdots, j_{1, t_{1}}\right\}, \cdots,\left\{j_{s, 1}, \cdots, j_{s, t_{s}}\right\}$ the collections of strictly glueable vertices of $Q^{\prime}$. then Aut $\left(\mathcal{A}_{Q^{\prime}}\right)$ is a subgroup of Aut $\left(\mathcal{A}_{Q}\right) \times S_{t_{1}} \times \cdots \times S_{t_{s}}$, and $A u t^{+}\left(\mathcal{A}_{Q^{\prime}}\right)$ is a subgroup of $A u t^{+}\left(\mathcal{A}_{Q}\right) \times S_{t_{1}} \times \cdots \times S_{t_{s}}$.

Proof. (a) We define a group homomorphism $S_{\mathcal{A}}$ from $\operatorname{Aut}\left(\mathcal{A}_{Q^{\prime}}\right)$ to $\operatorname{Aut}\left(\mathcal{A}_{Q}\right)$, and prove the following diagram of group homomorphisms commutes :

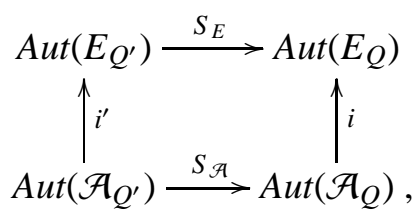

where $i$ and $i^{\prime}$ are injections by Theorem 3.14 a), and $S_{E}$ is the isomorphism given in subsection 3.2. Then the morphism $i \circ S_{\mathcal{A}}=S_{E} \circ i^{\prime}$ is injective, thus $S_{\mathcal{A}}$ is injective, that is, $\operatorname{Aut}\left(\mathcal{A}_{Q^{\prime}}\right)$ is a subgroup of $\operatorname{Aut}\left(\mathcal{A}_{Q}\right)$.

We consider the rooted cluster algebra $\left(Q^{\prime}, \mathbf{x}^{\prime}, \mathcal{A}_{Q^{\prime}}\right)$ and the rooted cluster algebra $\left(Q, \mathbf{x}, \mathcal{A}_{Q}\right)$. Assume that $\mathbf{x}=\left\{x_{1}, x_{2}, \cdots, x_{n}\right\}$ and $\mathbf{x}^{\prime}=\left\{x_{1}, x_{2}, \cdots, x_{n}, x_{n+1}, \cdots, x_{n+m}\right\}$, where $x_{n+1}, \cdots, x_{n+m}$ are frozen variables of $\mathcal{A}_{Q^{\prime}}$. Define $\Phi: \mathcal{A}_{Q^{\prime}} \rightarrow \mathcal{A}_{Q}$ as the specialization, that is, specializing frozen variables in $\mathcal{A}_{Q^{\prime}}$ to the integer 1 . It is clearly that $\Phi\left(\mathbf{x}^{\prime}\right)=\mathbf{x} \sqcup\{1\}$. It is proved in 
[CZ14](Proposition 2.39) that $\Phi$ is a surjective rooted cluster morphism from $\left(Q^{\prime}, \mathbf{x}^{\prime}, \mathcal{A}_{Q^{\prime}}\right)$ to $\left(Q, \mathbf{x}, \mathcal{A}_{Q}\right)$. Let $f$ be an element in $\operatorname{Aut}\left(\mathcal{A}_{Q}\right)$. We define $S_{\mathcal{A}}(f)=\Phi f \Phi^{-1}: \mathcal{A}_{Q} \rightarrow \mathcal{A}_{Q}$, then it is a ring homomorphism. To prove that $S_{\mathcal{A}}(f)$ is an cluster automorphism on $\mathcal{A}_{Q}$, we show that it satisfies the conditions in Proposition 2.5 (2). This easily follows from that $f$ is a cluster automorphism. Moreover, it is not hard to see that $S_{\mathcal{A}}$ is a group homomorphism. Finally, the above diagram is commutative since $S_{E}$ and $S_{\mathcal{A}}$ are both defined by specializations. Then $\operatorname{Aut}\left(\mathcal{A}_{Q^{\prime}}\right)$ is a subgroup of $\operatorname{Aut}\left(\mathcal{A}_{Q}\right)$. The injection $S_{\mathcal{A}}: \operatorname{Aut}\left(\mathcal{A}_{Q^{\prime}}\right) \rightarrow \operatorname{Aut}\left(\mathcal{A}_{Q}\right)$ induces an injection from $A u t^{+}\left(\mathcal{A}_{Q^{\prime}}\right)$ to $A u t^{+}\left(\mathcal{A}_{Q}\right)$. Thus we have done.

(b) This follows from Theorem 3.14(b) and (a).

Corollary 3.17. (a) For a cluster algebra with principal coefficients, excepting type $A_{1}$, its cluster automorphism group is a subgroup of the cluster automorphism group of its principal part cluster algebra.

(b) For a universal geometric cluster algebra, excepting type $A_{1}$, its cluster automorphism group is a subgroup of the cluster automorphism group of its principal part cluster algebra.

Proof. It is prove in Proposition 3.8 and Proposition 3.9 that these two kinds of cluster algebras are prime gluing free, and thus gluing free. Then the result follows from above theorem.

Example 7. We consider the cluster algebra $\mathcal{A}_{Q}$ of type $A_{2}$ and the two of its universal geometric cluster algebras $\mathcal{A}_{Q^{\prime}}$ and $\mathcal{A}_{Q^{\prime \prime}}$ in Example 1 Then the cluster automorphism groups Aut $\left(\mathcal{A}_{Q^{\prime}}\right)$ and $\operatorname{Aut}\left(\mathcal{A}_{Q^{\prime \prime}}\right)$ are both subgroups of $\operatorname{Aut}\left(\mathcal{A}_{Q}\right)$ which is isomorphic to $D_{5}$. A straightforward compute shows that $\operatorname{Aut}\left(\mathcal{A}_{Q^{\prime}}\right) \cong D_{5}$ and $\operatorname{Aut}\left(\mathcal{A}_{Q^{\prime \prime}}\right)=\{$ id $\}$. We prove in the subsequent paper $[C Z 15 a]$ that $\operatorname{Aut}(\mathcal{A})$ is always isomorphic to Aut $\left(\mathcal{A}^{\text {univ }}\right)$ for a cluster algebra $\mathcal{A}$ of finite type and its FZ-universal cluster algebra $\mathcal{A}^{\text {univ }}$.

For a gluing free cluster algebra, its cluster automorphism group may be a proper subgroup of the cluster automorphism group of its principal part cluster algebra (see Example 6 and Example 77. We show in the following theorem that for most cluster algebras associated to surfaces, the coefficients do not affect the cluster automorphism groups.

Theorem 3.18. Let $(S, M)$ be an oriented marked Riemann surface satisfies the Assumption 1, and not be a 4-gon. Let $\mathcal{A}(S, M)$ and $\mathcal{A}^{b}(S, M)$ be the cluster algebras associated to $(S, M)$ without coefficients and with coefficients respectively. Then there are isomorphisms $A u t^{+}(\mathcal{A}(S, M)) \cong$ $A u t^{+}\left(\mathcal{A}^{b}(S, M)\right)$ and $\operatorname{Aut}(\mathcal{A}(S, M)) \cong \operatorname{Aut}\left(\mathcal{A}^{b}(S, M)\right)$.

Proof. For the surface satisfies the Assumption 1, its marked mapping class group $\mathcal{M G} \mathcal{G}_{\bowtie}(S, M)$ are isomorphic to the direct cluster automorphism group $A u t^{+}(\mathcal{A}(S, M))$. On the one hand, it follows from Proposition 3.10 and Theorem 3.16 that $\mathrm{Aut}^{+}\left(\mathcal{A}^{b}(S, M)\right)$ is a subgroup of $A u t^{+}(\mathcal{A}(S, M))$, and thus a subgroup of $\mathcal{M G} \mathcal{G}_{\bowtie}(S, M)$. On the other hand, it is clearly that an element in the marked mapping class group $\mathcal{M G}_{\bowtie}(S, M)$ maps the boundaries to the boundaries and maintains the combinatorics of the tagged triangulations with boundaries. Thus it not only induces a cluster automorphism of $\mathcal{A}(S, M)$, but also induces a cluster automorphism of $\mathcal{A}^{b}(S, M)$. Therefore the three groups $A u t^{+}(\mathcal{A}(S, M)), \mathcal{M G} \mathcal{G}_{\bowtie}(S, M)$ and $A u t^{+}\left(\mathcal{A}^{b}(S, M)\right)$ are isomorphic. For the second isomorphism, note that if $\operatorname{Aut}^{+}(\mathcal{A}(S, M)) \cong \operatorname{Aut}(\mathcal{A}(S, M))$ then $\operatorname{Aut}(\mathcal{A}(S, M)) \cong \operatorname{Aut}\left(\mathcal{A}^{b}(S, M)\right)$. Now we assume that $A u t^{+}(\mathcal{A}(S, M))$ is a proper subgroup of $\operatorname{Aut}(\mathcal{A}(S, M))$, then by Lemma 2.6 
its index is 2. Thus there exist two triangulations $T$ and $T^{\prime}$ of $(S, M)$ such that $Q_{T} \cong Q_{T^{\prime}}^{o p}$. Then by the orientation and the combinatorics of the surface, we have $Q_{T^{b}} \cong Q_{T^{\prime b}}^{o p}$. Thus the index of $A u t^{+}\left(\mathcal{A}^{b}(S, M)\right)$ in $\operatorname{Aut}\left(\mathcal{A}^{b}(S, M)\right)$ is also 2. On the other hand, there is an isomorphism $A u t^{+}(\mathcal{A}(S, M)) \cong A u t^{+}\left(\mathcal{A}^{b}(S, M)\right)$ from the first step, and $\operatorname{Aut}\left(\mathcal{A}^{b}(S, M)\right)$ is a subgroup of $\operatorname{Aut}(\mathcal{A}(S, M))$ by Theorem 3.16, Thus we have an isomorphism between $\operatorname{Aut}(\mathcal{A}(S, M))$ and $\operatorname{Aut}\left(\mathcal{A}^{b}(S, M)\right)$.

Theorem 3.19. Let $Q$ be a quiver with at least two vertices. Let $\mathcal{A}^{p r}$ be the principal coefficient cluster algebra of $Q$. Then $A u t\left(\mathcal{A}^{p r}\right) \cong A u t^{+}\left(\mathcal{A}^{p r}\right) \cong A u t(B) \cong A u t\left(B^{p r}\right)$.

Proof. Firstly, note that $A u t(B) \cong A u t\left(B^{p r}\right)$, and an automorphism of $B$, or equivalently, an automorphism of $B^{p r}$ induces an automorphism of $\mathcal{A}^{p r}$ by exchanging the initial cluster variables of $\mathcal{A}^{p r}$. Thus $A u t(B) \cong A u t\left(B^{p r}\right) \subseteq A u t\left(\mathcal{A}^{p r}\right)$. Secondly, it is proved in [BDP14] (see Proposition 2.10 and Corollary 2.12) that in seeds on exchange graph $E_{\mathcal{A} p r}$, excepting $Q^{p r}$ itself, there exist no ice quivers which are isomorphic to $Q^{p r}$, and there exist no ice quivers which are isomorphic to the opposite quiver of $Q^{p r}$. Thus any automorphism $\sigma \in A u t\left(\mathcal{A}^{p r}\right)$ is direct and must map $Q^{p r}$ to itself. Therefore $\operatorname{Aut}\left(\mathcal{A}^{p r}\right) \cong A u t^{+}\left(\mathcal{A}^{p r}\right) \cong A u t(B) \cong A u t\left(B^{p r}\right)$.

\section{Acknowledgements}

The authors wish to thank Dong Yang, Wuzhong Yang, Jie Zhang and Yu Zhou for helpful discussions on the topic, and thank Bangming Deng, Yang Han, Yanan Lin, Liangang Peng and Jie Xiao for valuable comments. The first author wish to thank Thomas Brüstle for useful discussions during a conference [B14] in August 2014.

\section{References}

[ADS14] Assem I, Dupont G, Schiffler R. On a category of cluster algebras. Journal of Pure and Applied Algebra, 2014, 218(3):553-582.

[ASS12] Assem I, Schiffler R, Shramchenko V. Cluster automorphisms. Proceedings of the London Mathematical Society, 2012, 104(6):1271-1302.

[ASS13] Assem I, Schiffler R, Shramchenko V. Cluster automorphisms and compatibility of cluster variables. Glasgow Mathematical Journal, 2014, 56(03):705-720.

[BD13] Blanc J D, Dolgachev I. Automorphisms of cluster algebras of rank 2. Transformation Groups, 2015, 20(1):1C20.

[BDP14] Brstle T, Dupont G, Protin M. On maximal green sequences. International Mathematics Research Notices, 2014, 2014(16): 4547-4586.

[BIRS09] Buan A, Iyama O, Reiten I, Scott J. Cluster structure for 2-Calabi-Yau categories and unipotent groups. Compositio Mathematica, 2009, 145(4):1035-1079.

[B14] Brüstle T. Private communication. International Conference on Representation theory of Algebras (Sanya China), 2014. 
[BQ12] Brüstle T, Qiu Yu. Tagged mapping class groups: Auslander-Reiten translation. Mathematische Zeitschrift, 2015, 279(3-4):1103C1120.

[BS13] Bridgeland T, Smith I. Quadratic differentials as stability conditions. Publications mathmatiques de l'IHS, 2013:1-124.

[CZ14] Wen Chang, Bin Zhu. On rooted cluster morphisms and cluster structures in 2-CalabiYau triangulated categories, arXiv:1410.5702 (2014).

[CZ15a] Wen Chang, Bin Zhu. Cluster automorphism groups of cluster algebras of finite type.

[CZ15b] Wen Chang, Bin Zhu. Cluster automorphism groups and automorphism groups of exchange graphs.

[DWZ10] Derksen H, Weyman J, Zelevinsky A. Quivers with potentials and their representations II: Applications to cluster algebras. Journal of the American Mathematical Society, 2010, 23(3): 749-790.

[F10] Fomin S. Total positivity and cluster algebras. Proceedings of the International Congress of Mathematicians. Volume II. Hindustan Book Agency, New Delhi, 2010:125C145.

[FST08] Fomin S, Shapiro M, Thurston D. Cluster algebras and triangulated surfaces. Part I: Cluster complexes. Acta Mathematica, 2008, 201:83-146.

[FZ02] Fomin S, Zelevinsky A. Cluster algebras. I. Foundations. Journal of the American Mathematical Society, 2002, 15(2), 497-529.

[FZ03] Fomin S, Zelevinsky A. Cluster algebras. II. Finite type classification. Inventiones Mathematicae, 2003, 154(1):63-121.

[FZ07] Fomin S, Zelevinsky A. Cluster algebras IV: Coefficients. Compositio Mathematica, 2007, 143:112-164.

[CKLP13] Irelli C G, Keller B, Labardini-Fragoso D, Plamondon P G. Linear independence of cluster monomials for skew-symmetric cluster algebras. Compositio Mathematica, 2013, 149(10):1753-1764.

[G14] Gratz S. Cluster algebras of infinite rank as colimits. arXiv:1410.5374, 31 pages.

[GSV08] M. Gekhtman, M. Shapiro and A. Vainshtein, On the properties of the exchange graph of a cluster algebra, Math. Res. Lett, 15(2), (2008), 321-330.

[GLS08] Gekhtman M, Shapiro M, Vainshtein A. On the properties of the exchange graph of a cluster algebra. Mathematical Research Letters, 2008, 15(2):321-330.

[K12] Keller B. Cluster algebras and derived categoreis. arXiv:1202.4161, 60 pages.

[KP13] King A, Pressland M. Labelled Seeds and Global Mutations. arXiv:1309.6579, 24 pages.

[L10] Leclerc B. Cluster algebras and representation theory. Proceedings of the International Congress of Mathematicians. Vol. IV, Hindustan Book Agency, New Delhi, 2010:24712488 . 
[M14] Marsh R J. Lecture Notes on Cluster algebras. Zurich Lectures in advanced Mathematics, European Mathematical Society Publishing house, 2014.

[N13] Ndoune N. On involutive cluster automorphisms. arXiv:1306.6322, 16 pages.

[R10] Reiten I. Cluster categories. Proceedings of the International Congress of Mathematicians. Volume I, Hindustan Book Agency, New Delhi, 2010:558C594.

[R12] Reading N. Universal geometric coefficients for the once-punctured torus. arXiv:1212.1351, 26 pages.

[R14a] Reading N. Universal geometric cluster algebras. Mathematische Zeitschrift, 2014, 277(1-2):499C547.

[R14b] Reading N. Universal geometric cluster algebras from surfaces. Transactions of the American Mathematical Society, 2014, 366(12):6647C6685.

[ST09] Schiffler R, Thomas H. On cluster algebras arising from unpunctured surfaces. International Mathematics Research Notices. IMRN, 2009, 17:3160C3189.

[S10] Saleh I. Exchange Automorphisms of Cluster Algebras. arXiv:1011.0894 12 pages.

[Z06] Bin Zhu. Applications of BGP-reflection functors: isomorphisms for cluster algebras. Science in China. Series A. Mathematics, 2006, 49(12):1839-1854. 